SLAC-PUB-I06 ReV.

TTP- 169

May 1965

UNTTARY GROUPS: REPRESENTATTONS AND DECOMPOSITIONS*

\begin{abstract}
c. Itzrkisoni:
Stanford Iinear Accelerator Center, Stanford University, Stanford, California

and

M. Nauenberg ${ }^{\ddagger}$

Physics Department, Stanford University, Stanford, California
\end{abstract}

(Submitted to Review of Modern Physics)

\footnotetext{
*Work supported by the U. S. Atomic Energy Commission and by the U. S. Air Force through Air Force office of Scientific Research Contract AF 49(638)-1389.

ton leave from Service de Physique Theorique, CEN Saclay, BP No. 2, Gif sur Yvette (S. et 0. ), France

\#. P. Sloan Fellow
} 


\section{TABIE OF CONTEINTS}

Page

I. Introduction . . . . . . . . . . . . . . . . . . I

II. Revicw of uni ury grouss . . . . . . . . . . . . . 2

III. Description of the irreducible representations of $S_{n} \cdot \cdot \cdot 8$

IV. The generators of $\mathrm{SU}_{n} \cdot$. . . . . . . . . . . . . . 19

V. Decomposition of the product of two representations of $\mathrm{SU}_{\mathrm{n}} \cdot \quad \geq 6$

VI. The $\left(\mathrm{SU}_{\mathrm{m}}, \mathrm{SU}_{\mathrm{n}}\right)$ content of irredncibie representations of

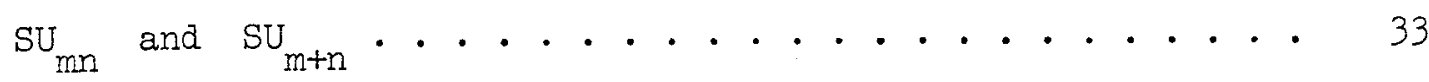

VII. Tables ...................... . . 41

Appendix: The symmetric group and properties of the Young symmetry operators . . . . . . . . . . . . 67 
I. INTRODUCTION

In these notes we describe some fundamental properties of the irreducible representations of $\mathrm{SU}_{\mathrm{n}}$, the special unitary group in $\mathrm{n}$-dimensions. We use, as basis for these representations, tensors which satisfy certain symmetry properties witi res ect to ker..ut iticiss of their indices, and discuss briefly in this connection the symmetric group. We also relate this global analysis of the representations to the method based on the infinitesmial transformations of continuous groups: the Lie algebra of $\mathrm{SU}_{\mathrm{n}}$.

The unitary groups are very important in physics. The best-known example is $\mathrm{SU}_{2}$ which describes the spin and isospin of particles. Recently unitary groups in higher dimensions have been applied with success to study the properties of elementary particles. Although the mathematical theory of these groups and their representations has been developed for a long time, useful results are somewhat scattered in the literature. We therefose have attempted to collect here some formulas and tricks, and have computed several tables that are useful in the application of unitary groups to particle physics.

Throughout the text we have tried to give some idea of how one derives the more important results; this should help the reader to remember them and also serve to explain our notation. Some topics which we have left out of our discussion include the construction of explicit basis in each representation space in terms of which to express the analogs of Clebsch-Gordan coefficients, and formulas for the elements of the representation matrices. There is no special reason for such omissions which are useful in practical applications. However, for low dimensional representations the tensor 
methods which are described here can be successfully used. The discussion of $\mathrm{SU}_{\mathrm{n}}$ can be extended with minor modifications to the special linear groups $S L(n, R)$ and $S L(n, C)$, the groups of $n \times n$ matrices of determinant one with real and complex entries respectively.

We have included a short list of books to which we refer for omitted proofs, and some recent articles on the subieci.

\section{REVIEW OF UNITARY GROUPS}

When dealing with symmetries in particle physics, one is led to study the representations of some simple groups. Here we are concerned mainly with the special unitary groups in $n$ limensions, denoted by $\mathrm{SU}_{\mathrm{n}}$. To be precise, our group is the set of $n \times n$ matrices with complex entries which are unitary and of determinant equal to one. A typical such matrix will be denoted by $g$. By a unitary transformation (which can be chosen to be of determinant one) such a matrix can be diagonalized; hence, for a given $g$ there always exists a $g^{\prime}$ in the group, such that

$$
g^{\prime} g^{\prime}{ }^{-1}=\left(\begin{array}{lllll}
\epsilon_{1} & & & & \\
& & \cdot & & \\
& & & \cdot \epsilon_{n}
\end{array}\right)
$$

where the $\epsilon_{I}$ are just the eigenvalues of $g$ of modulus one, and $\epsilon_{12} \epsilon_{2} \cdot \cdot \epsilon_{n}=1$. Any unitary matrix can be written

$$
g=e^{i h}
$$

where $h$ is a hermitian matrix. This is an immediate consequence of Eq. (I). Moreover, for $g$ to have determinant one, it is sufficient that $h$ be traceless. Now an arbitrary hermitian matrix is given in terms of the $n$ diagonal elements which are neoessarily real, and the $\frac{n(n-1)}{2}$ complex elements 
above the main diagonal; hence, this matrix depends on $n^{2}$ real-parameters. If we impose the condition that the trace be zero, we are left with $n^{2}-1$ independent parameters.

The group $\mathrm{SU}_{\mathrm{n}}$ has three fundamental properties:

(I) It is compact. The precise meaning of this word in this context is the following: If we are given $a_{11}$ irfinits seginence of elements $g_{1} \cdots g_{i} \cdots$, we can always extract a subsequence which converges to an element of the group.

We shall not investigate further the topological properties, but mainly remark that the compactness property has the important consequence that the irreducible representations to be introduced below enjoy the following properties: (i) They are all finite dimensional.

(ii) They are all equivalent to unitary representations.

(iii) Any representation can be split in a airect sum of irreducible representations.

(2) $\mathrm{SU}_{\mathrm{n}}$ is a Iie group. This means that certain differentiability conditions (obvious in this case) are satisfied. This reduces the study of such a group to the study of the so-called infinitesimal elements, i.e., those close to unity. We discuss briefly this approach in Section IV; however, we shall not emphasize this point of view.

(3) Finally, $\mathrm{SU}_{\mathrm{n}}$ is a simply connected group. Connected means that, given an arbitrary element $g$, one can find a continuous set of elements in the group $g(t)$, where $0 \leq t \leq I$ such that $g(0)$ is the identity $e$, and $g(1)=g$. In a simply connected group two such "paths" leading from e to $g$ can be continuously transformed in one another. In summary:

$\mathrm{SU}_{\mathrm{n}}$ is a simply connected compact lie group depending on $\mathrm{n}^{2}-1$ real parameters. 
In view of what has been said we need only define representations in finite dimensional spaces. This is always understood here. By representation of a group $G$ one means a correspondence which assigns to every element $g$ a linear operator $A(g)$ (i.e., a matrix once a basis has been chosen) in some vector space, the carrier of representation, such that the image of $e$ is the ilentity operster $T$, and the group law is preserved; i.e.,

$$
A(g) A\left(g^{\prime}\right)=A\left(g g^{\prime}\right)
$$

The carrier space is assumed to be a complex vector space, i.e., the matrices $A(g)$ have complex entries.

Two representations are equivalent if the carrier spaces can be put in a one-to-one linear correspondence $\mathrm{x} \leftrightarrow \mathrm{x}^{\prime}$ with the property that $A(g) x \leftrightarrow A^{\prime}(g) x^{\prime}$. In the following we shall be concerned with representations up to equivalence; i.e., we shall identify equivalent representations. If a basis has been chosen in the two equivalent carrier spaces, and if $A$ and $A^{\prime}$ denote the matrices of the representations, the statement of equivalence can be rephrased by saying that there exists a non-singular matrix $B$ such that for every $g$ in the group

$$
A^{\prime}(g)=B A(g) B^{-1}
$$

A subspace of the carrier space is said to be invariant if it is left unchanged by all operators $A(g)$. The representation is said to be reducible if such a proper invariant subspace exists; otherwise, it is called irreducible. In our case, $\left[G=S_{n}\right]$ reducibility implies, in fact, a little more, namely, if there exists a proper invariant subspace, then one can find a complementary subspace which is also invariant. In other words, the representation splits. In pictures, if all the matrices $A(g)$ 
have the form

$$
\left(\begin{array}{l|l}
x & x \\
\hline 0 & x
\end{array}\right) \text {, }
$$

there exists a basis in which $A(g)$ takes the form

$$
\left(\begin{array}{l|r}
x & 0 \\
\hline 0 & x
\end{array}\right)
$$

we say that the representation is completely reducible. Given a representation we can thus split it again and again until we reach irreducible parts.

Given an irreducible representation $A(g)$, the only linear operators $C$ which commute with every $\mathrm{A}(\mathrm{g})$, i.e., $\mathrm{CA}(\mathrm{g})=\mathrm{A}(\mathrm{g}) \mathrm{C}$ for all $\mathrm{g}$, are multiples of the identity $\mathrm{C}=\lambda I$ (Schur's Lema). The converse is also true.

Our first task will be to describe all the irreducible representations of $\mathrm{SU}_{\mathrm{n}}$ up to equivalence. This construction is entirely algebraic in nature, and is carried out in the next section. However, since the results are often given an interesting meaning using some analytic tools, we say a word on characters and integration on the group.

Given a representation $\mathrm{A}(\mathrm{g})$, we can compute the trace $\chi(\mathrm{g}) \equiv \sum_{i} A_{i i}(\mathrm{~g})$ which is basis independent. The (complex valued) function $g \rightarrow x(g)$ is the character of the representation. Immediate properties are*

$$
\begin{aligned}
x\left(g^{\prime} g g^{\prime-1}\right) & =x(g) \\
x\left(g^{-1}\right) & =\bar{x}(g)
\end{aligned}
$$

The second property stems from the fact that every representation of the compact group $\mathrm{SU}_{\mathrm{n}}$ is equivalent to a unitary representation. The importance of the characters lies in the fact that it determines the representation up

\footnotetext{
${ }^{*}$ A bar over a number means complex conjugation
} 
to equivalence: i.e., two representations with the same characters are equivalent. Using Eqs. (I) and (3), one obtains the result that $x(g)$ is in fact a symmetric function of $\epsilon_{1} \cdot \cdot \epsilon_{n}$ where $\epsilon_{1} \cdot \cdot \epsilon_{n}$ are the eigenvalues of $g$. Stated in an equivalent manner, $x(g)$ is a function of the coefficients $\left(a_{1} \cdot a_{n}\right)$ of the chararterj tic rolyncis of 8 :

$$
\operatorname{det}(1-\lambda g)=\sum_{p=1}^{n}(-1)^{p} a_{p} \lambda^{p} \quad a_{0} \equiv 1
$$

(In fact $a_{n}$ is also equal to one, since det $g=1$. ) Now it is possible to introduce an invariant integration on $G \equiv S U_{n} \cdot$ By this we mean the following: parameterize in some way the group (in our case with $n^{2}-1$ real parameters); then there exists a measure $d_{\mu}(g)$ on the group, such that if $g^{\prime}$ is a fixed element in the group,

$$
d \mu\left(g^{\prime} g\right)=d \mu(g)
$$

and

$$
d \mu\left(g g^{\prime}\right)=d \mu(g)
$$

Finally, $d \mu$ is essentially unique up to scale factor. We use this freedom and the compactness of $\mathrm{SU}_{\mathrm{n}}$ to normalize $\mu(\mathrm{g})$,

$$
\int_{G} d \mu(g)=1
$$

In the case of $\mathrm{SU}_{\mathrm{n}}$ it turns out that a particular choice of parameters is indicated. Let us go back to Eq. (l) and put $\epsilon_{i}=e^{i 2 \pi \varphi i}$. Then it is possible to make the parametrization in such a way that

$$
\begin{gathered}
\mathrm{d \mu}(\mathrm{g})=\frac{\delta\left(\Sigma \varphi_{i}\right)}{\Omega} \prod_{i \neq j}\left|\left(\epsilon_{i}-\epsilon_{j}\right)\right| \mathrm{d} \varphi_{I} \mathrm{~d} \varphi_{2} \cdot \cdots \cdot d \varphi_{n} \mathrm{~d} \omega_{\mathrm{g}} \\
-6-
\end{gathered}
$$


where $d \omega_{g}$ depends essentially on the matrix which diagonalizes $g$ and need not be considered further here, and $\Omega$ is a normalization constant. Note that

$$
\pi_{i f j}\left|\left(\varepsilon_{i}-\epsilon_{j}\right)\right|=\Delta(\epsilon) \bar{\Delta}(\epsilon)
$$

with

$$
\Delta(\epsilon)=\pi_{i<j}\left(\epsilon_{i}-\epsilon_{j}\right)
$$

We shall denote the invariant measure $\mathrm{d} \mu(\epsilon)$

$$
d \mu(\epsilon)=\frac{\bar{\Delta}}{\Omega} \phi\left(\Sigma \varphi_{i}\right) d \varphi_{1} \cdot \cdot d \varphi_{n}
$$

with $\Omega$ determined by the condition

$$
\int_{\substack{0 \leq \varphi_{1} \leq 1 \\ 0 \leq \varphi_{2} \leq 1 \\ \ldots \ldots \ldots}} d \mu(\epsilon)=1
$$

The following important orthogonality relations hold. If $x^{i}(\epsilon)$ is the character of an irreducible representation of $\mathrm{SU}_{n}$, then

$$
\int \bar{x}(\epsilon) x(\epsilon) \mathrm{d} \mu(\epsilon)=1
$$

If $\chi(\epsilon)$ and $\chi^{\prime}(\epsilon)$ correspond to inequivalent irreducible representations

$$
\int \bar{x}(\epsilon) \dot{x}(\epsilon) \mathrm{d} \mu(\epsilon)=0
$$

Applications of these formulas will be found in Section III. 
III. DESCRIPTION OF THE IRREDUCIBLE REPRESENTATIONS OF $\mathrm{SU}_{\mathrm{n}}$

We consider the set of tensors, $T_{i_{2} i_{2} \ldots i_{f}}$, where the indices $1_{1}, i_{2}, \ldots i_{1}$, run from 1 to $n$. To each unitary matrix $g$, we associate a linear transformation $A(g)$ in the space of tensors

$$
T_{i_{1}} \ldots i_{f} \rightarrow{ }_{i_{1}} \ldots i_{f}=\Delta(g)_{i_{1}} \ldots i_{f} ; i_{1}^{i} \ldots i_{f} T_{i_{1}^{\prime}} \ldots i_{f}^{\prime}
$$

where a sum over repeated indices is implied, and

$$
A(g)_{i_{1}} \cdots i_{f} ; i_{1}^{\prime} \ldots i_{f}^{\prime} \equiv g_{i_{1}}{ }^{\prime} \cdots g_{i_{f} i_{f}^{\prime}} \cdot \cdots
$$

In a more compact notation,

$$
A(g)=g \times g \times \cdots \times g
$$

which defines $\mathrm{A}(\mathrm{g})$ as the Kronecker or direct product of matrices $\mathrm{g}$.

The matrices $A(g)$ build a unitary, but in general reducible representation of $\mathrm{SU}_{\mathrm{n}}$. They satisfy the important property that they are bisymmetric, that is, invariant under a permutation of the indices $i_{1} \ldots i_{f}$ and the same permutation on the indices $i_{I}^{\prime} \cdot{ }^{\prime} i_{f}^{\prime}$. A permutation $p$ on $f$ integers is denoted by

$$
P=\left(\begin{array}{llll}
1 & 2 & \cdots & f \\
p_{1} & p_{2} & & \\
p_{f}
\end{array}\right)
$$

where $p_{1} p_{2} \cdots p_{f}$ is a rearrangement of the ordered $f$ integers, and the $p$ permutation of the indices is indicated by

$$
p\left(i_{1} \cdots i_{f}\right)=\left(i_{p_{1}} i_{p} \cdots i_{p}\right)
$$


or more briefly $p(i)=\left(i_{p}\right)$. The property of bisymmetry of $A(g)$ is then expressed by the relation

$$
A(g)\left(\dot{i}_{p}, i_{p}^{\prime}\right)=A(g)\left(i, i^{\prime}\right)
$$

It can be readily seon that if we tale a Iinear combination of tensors satisfying some symmetry condition with respect to the permutation of their indices, this property is preserved under the transformations generated by $A(g)$. In general, these symmetrized tensors span a subspace of the tensor space which is then invariant under $\mathrm{SU}_{n}$, and therefore gives a representation of the group. The fundamental theor:m on representations of unitary groups states that there exists maximal symmetry conditions which can be imposed on the tensors, such that the resulting invariant subspaces generate all the irreducible representations of $\mathrm{SU}_{\mathrm{n}} \cdot{ }^{*}$

We begin by giving a description of these maximal symetry conditions : by means of Young tableaux. A Young tableau consists of an array of $f$ boxes with $f_{1}$ boxes in the first row, $f_{2}$ boxes in the second row, and $f_{n-1}$ boxes in the $n-1-t h$ row, where the integers $f_{1}, f_{2} \ldots f_{n-1}$ satisfy the relations

$$
f_{1} \geq f_{2} \geq f_{3} \geq \cdots \geq f_{n-1}
$$

and

$$
f=f_{1}+f_{2}+\cdots f_{n-1}
$$

For convenience of notation we include in some formulas $f_{n}=0$.

* See Reference 1, Chapters III and IV for the full development of this duality between the linear groups and the symmetric groups. 
In pictures, a tableau is usually drawn as follows:

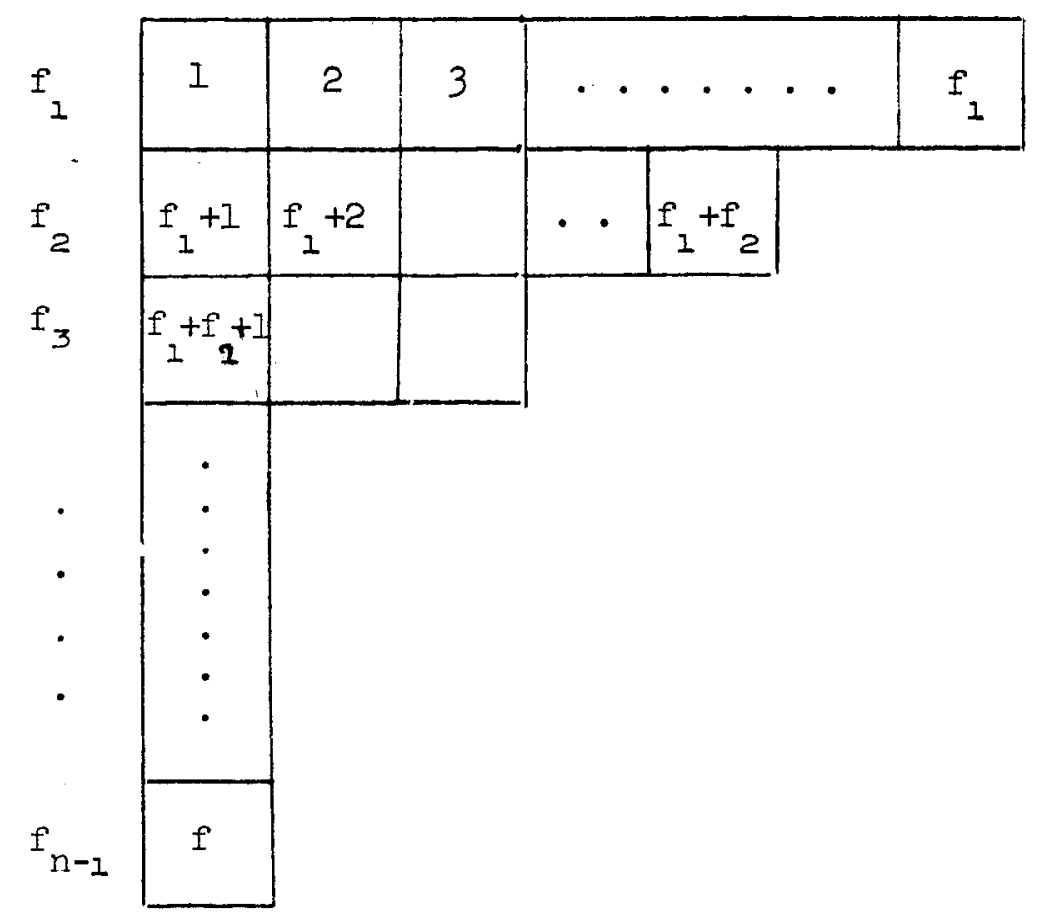

To this tableau corresponds the following symmetry operation on a tensor $T_{i_{1}} \ldots i_{f}$

(i) Symmetrize completely with respect to the first $f_{I}$ indices $i_{I} \ldots i_{f_{I}}$, the following $f_{2}$ indices $i_{f_{1}+1} \cdots i_{f_{1}}+f_{2}$, and so on, thus getting a tensor

$$
T i_{1} \cdots i_{f_{1}}, i_{f_{1}+1} \cdots i_{f_{1}+f_{2}}, \cdots
$$

(ii) Then antisymmetrize the tensor $\mathrm{T}^{\prime}$ with respect to the indices $i_{1}, i_{f_{1}+1}, i_{f_{1}+f_{2}+1} \cdots$, the indices $i_{2}, i_{f_{1}+2}, i_{f_{1}+f_{2}} \ldots$, and so on. The resulting set of tensors $T^{\prime \prime}$ form the basis of an invariant subspace which generates an irreducibie representation of $\mathrm{SU}_{\mathrm{n}}$. We can write in compact notation

$$
T_{i_{1}}^{\prime \prime} \ldots i_{f}=Y T_{i_{1}} \ldots i_{f}
$$

where

$$
Y=\Sigma \delta_{q} q p
$$

is the Young symmetry operator associated with the Young tableau. The 
sum in $\mathrm{Y}[\mathrm{Eq} .(5)]$, is carrled over all permutations $\mathrm{p}$ of integers in the same row, and all permutations $q$ of integers in the same column of the Young tableau, while $\delta_{q}$ is the signature of the permutation $q ; \delta_{q}=+l(-1)$ for $q$ even (odd). The tableau has no more than $n-1$ rows. This is a result of two facts: first, that it is impossible to antisymetrize more than $n$ indices each running from $I$ to $n$, and, second, that we restrict our attention to transformations of determinart $i .{ }^{*}$ To differen: ta? Jeaux correspond inequivalent representations.

There is a one-to-one correspondence between the Young tableaux of no more than $n-l$ rows and the irreducible representations of the group $S_{n}$.

The tableau with zero box corresponds to the identity representation, i.e., to the representation which assigns to every element of the group the unit operator in a one dimensional space, and will be denoted by a dot. The tableau with one box corresponds to the representation by the group itself. Among other interesting representation, let us point out the following:

(i) Representations with one row only, $f_{1}=f$. They correspond, according to what we have seen, to a carrier space of totally symmetric tensors. The dimension of this representation is easily computed as the number of ways one can choose $f$ objects among $n$ objects allowing repetitions, namely

$$
N=\left(\begin{array}{c}
n+f-1 \\
f
\end{array}\right) \equiv \frac{(n+f-1) !}{f !(n-1) !},
$$

(the familiar counting problem for an Einstein-Bose gas).

There is an infinite number of such representations.

${ }^{*}$ It is convenient to use the following convention. In some cases we add to a Young tableau of no more than $n-1$ rows, columns of $n$ boxes on the lef't. These new tableaux will be considered as equivalent to those where these extra columns are dropped. The dimension formula (see Eq. 10) is invariant under that transformation. 
(ii) Representations corresponding to rows of length 1 or 0 . In other words, the tableau is reduced to its first column. Excluding the identity representation, there are $n-1$ such representations

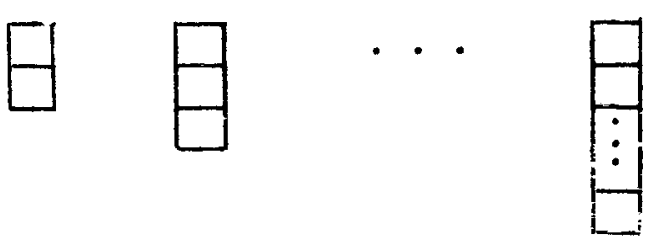

They correspond to carrier spaces built up of totally antisymetric tensors. If $\lambda$ is the length of the column, the representation is of dimension

$$
N=\left(\begin{array}{l}
n \\
\lambda
\end{array}\right)=\frac{\dot{n} !}{\lambda !(n-\lambda) !}
$$

(the counting problem for a Fermi-Dirac gas).

We shall give below a formula which gives the dimension of a general representation.

(iii) The representation with $f_{1}=2, f_{2}=1, f_{3}=1, \cdots \cdot f_{n-1}=1$. This is called the adjoint representation and is very important, because its basis transforms like the generators of the group. Let us briefly outline how one gets this representation. Let $\mathrm{h}$ be an arbitrary, traceless, $\mathrm{n} \times \mathrm{n}$ hermitian matrix. The set of these matrices is closed with respect to addition and multiplication by real numbers; hence, they build up a vector space whose dimension we have already computed to be $n^{2}-1$. The transformation

$$
h \rightarrow h^{\prime}=g h g^{-1}
$$

where $g$ is an element of $S_{n}$, is obviously a linear transformation of our set of hermitian matrices. We thus get a representation of $\mathrm{SU}_{\mathrm{n}}$ in this space which can be shown to be irreducible. This is the adjoint representation. 
Its dimension is

$$
N=n^{2}-1
$$

and with our choice of basis the representation consists of real matrices only.

(iv) Finaily, let us discurs cont,ag auji znt representations. Given any representation of a group by the correspondence

$$
g \rightarrow A(g)
$$

one can define the contragradient representation *

$$
g \rightarrow A^{T}\left(g^{-1} ;=A^{-2}(g)\right.
$$

One verifies that it is a representation, and also that it is reducible or not according to whether $A$ is reducible or not. If the representation $A$ is unitary, so is the contragradient representation which in fact is simply the complex conjugate of $A$, i.e., $g \rightarrow \bar{A}(g)$ in that case. Note in this connection that $\bar{T}_{i_{1}} \ldots i_{f}$, the complex conjugate of a tensor $T_{i_{2}} \ldots i_{f}$, transforms according to the rule $\overline{\mathrm{T}}^{\prime}=(\overline{\mathrm{g}} \times \overline{\mathrm{g}} \cdot \times \times \overline{\mathrm{g}}) \overline{\mathrm{T}}$ which is used to define contravariant tensors by setting the indices as superscripts, $T^{i_{I} \cdots i_{f}}$. The relation to covariant tensors is obtained through the Levi-Civita symbol $\epsilon_{i_{1} i_{2} \ldots i_{n}}$, which is totally antisymmetric in its $n$ indices, and equals +1 of - 1 according to whether $i_{1} i_{2} \ldots i_{n}$ is an even or oa permutation of the integers $1,2 \ldots n$. It can readily be seen that it is invariant under any unimodular transformations. For each contravariant index $i$, we multiply the contravariant tensor by $\epsilon_{i_{1} i_{2}} \ldots i_{n-1} i$ and sum over $i$ giving rise to $n-I$

\footnotetext{
*The superscript $T$ on $A$ Qenotes the transpose of $A$.
} 
covariant indices. For example, if $T^{i}$ is a contravariant tensor, of rank $I$, then

$$
T_{1} \ldots i_{n-1}=\epsilon_{i_{1}} \ldots i_{n-1} i T^{i}
$$

is a covariant antisymmetric tensor of rank $n-l$. Naturally, we can equally well construct contravariant indices from any covariant tensor which contains n-l antisymmetric incices. Fo example. if $A_{z_{2}} \ldots i_{n-i}$ is totally antisymmetric in $i_{1} \ldots \dot{i}_{n-1}$,

$$
y^{i}=\epsilon_{i i_{1}} \ldots i_{n-1} A_{i_{1}} \ldots i_{n-1}
$$

is a contravariant tensor. The raising and lowering of tensor indices by $\epsilon_{i_{I} \ldots i_{n}}$ makes it possible to contract these indices, e.g., the sum $\sum_{i} y_{i} y^{i}$ is an invariant.

If a representation is equivalent to a representation by real matrices, then it follows that it is equivalent to its contragradient. For a given tableau of $\mathrm{SU}_{\mathrm{n}}$ corresponding to a representation $\mathrm{A}$, one obtains the contragradient representation by the following process:

(i) Draw the initial Young tableau

(ii) Complete the drawing to obtain a rectangle of horizontal dimension $\mathrm{f}_{1}$ and vertical dimension $\mathrm{n}$.

(iii) The complementary part is the desired Young tableau if one rotates it by $\pi$. It is seen that the procedure is equivalent to saying that if $f_{1}^{\prime} \geq \cdots \geq f^{\prime}{ }_{n-1} \quad$ are the rows of the Young $t=b$ leau corresponding to the contragradient representation, then

$$
\begin{aligned}
f_{1}^{\prime} & =f_{1}-f_{n}=f_{1} \\
f_{2}^{\prime} & =f_{1}-f_{n-1} \\
f_{p}^{\prime} & =f_{1}-f_{n-p+1} \\
\vdots \cdot & \cdot \cdot \cdot \cdot \\
& -14-
\end{aligned}
$$


In particular, representations equivalent to their contragradient are such that

$$
f_{p}=f_{p}^{\prime}=f_{1}-f_{n-p+1}
$$

or

$$
f_{p}+f_{n-p+1}=f_{1} \quad p=I \ldots n
$$

As an example we see that the adjoint representation has this property. Obviously, a representation and its contragradient have the same dimensions; thence, the dimension formula has to be invariant with respect to the transformation $f_{p} \rightarrow f_{p}^{\prime}$

Digressing, we note that all the finite dimensional irreducible repre= sentations of the special linear group on real numbers $\mathrm{SL}(\mathrm{n}, \mathrm{R})$ can similarly be described in terms of the tensor spaces used for $\mathrm{SU}_{\mathrm{n}}$. The matrix elements of these representations are polynomials in the matrix elements of the element $g \in S L(n, R)$. If we extend these polynomials to complex values, we get a representation of the special linear group on complex numbers $\operatorname{SL}(n, C)$. The most general finite dimensional irreducible representations of this group are obtained by forming Kronecker products $D^{\prime} \times \bar{D}^{\prime \prime}$ where $D^{\prime}$ and $D^{\prime \prime}$ are representations of the type just discussed, and $\bar{D}^{\prime \prime}$ is the complex conjugate of $D^{\prime \prime}$.

As an example, the finite dimensional representations of $\operatorname{SI}(2, C)$. which is in two-to-one correspondence with the Iorentz group, can be labeled by two Young tableaux consisting of one row of $2 j_{1}$ and $2 j_{2}$ boxes respectively. It must be emphasized that $S L(n R)$ and $S I(n C)$ are not compact and that: the finite dimensional representations are not unitary. In order to find unitary representations one has to introduce infinite dimensional Hilbert spaces, which we shall not discuss here. 
We return to $\mathrm{SU}_{\mathrm{n}}$ and discuss the characters and dimensions of the representations.

We have already quoted the fact that a representation is completely determined by its character. The following formula* gives the character for the representation belonging to the Young cableau $f_{1}, f_{2} \ldots, f_{n}(=0)$ as a oymmetric function of the ejge valias $\epsilon_{2} \ldots \epsilon_{n}$ of the general element $g$ in $\mathrm{SU}_{\mathrm{n}}$ (Weyl's character formula)

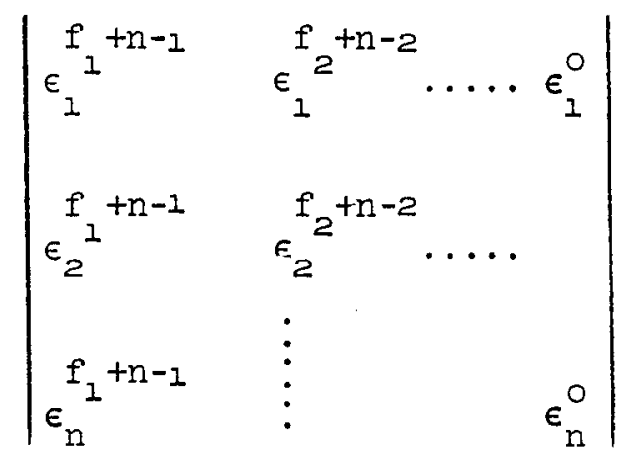

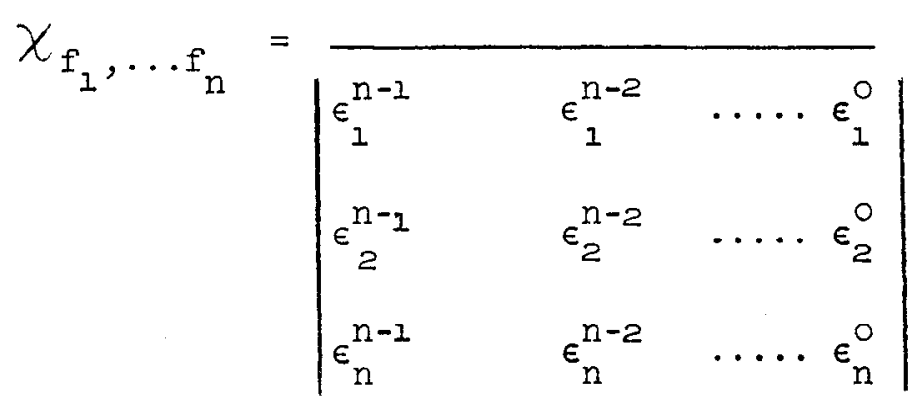

From thisformula one gets the dimension $N$ by letting $\epsilon_{1}, \ldots, \epsilon_{n}$ go to one, i.e., $\mathbb{N}$ is the character of the identity. The calculation must be made carefully because the denominator and the numerator vanish in this limit.

We set $l_{1}=f_{1}+n-1, l_{2}=f_{2}+n-2, \cdots$. In order to take a proper limit we first relax the condition $\epsilon_{I} \ldots \epsilon_{n}=1$ and choose

$$
\epsilon_{1}=\epsilon^{n-1} \quad \epsilon_{2}=\epsilon^{n-2} \ldots \quad \epsilon_{n}=\epsilon^{0}
$$

* See for instance Reference 1, page 201 . 
With $\epsilon \rightarrow e^{i \Phi}$, and $\Phi \rightarrow 0$, we have

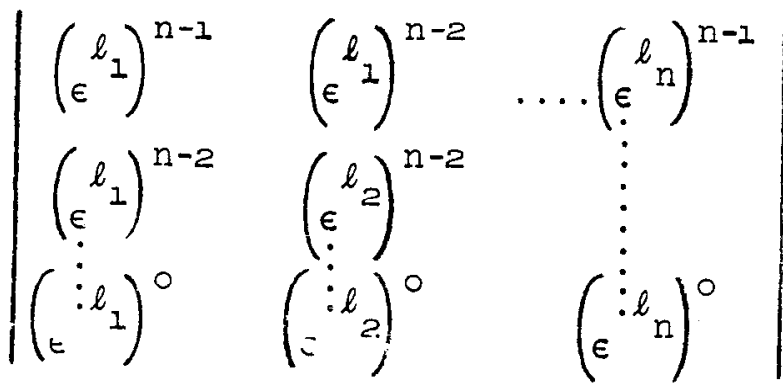

$$
\begin{aligned}
& N=\lim _{\Phi \rightarrow 0}
\end{aligned}
$$

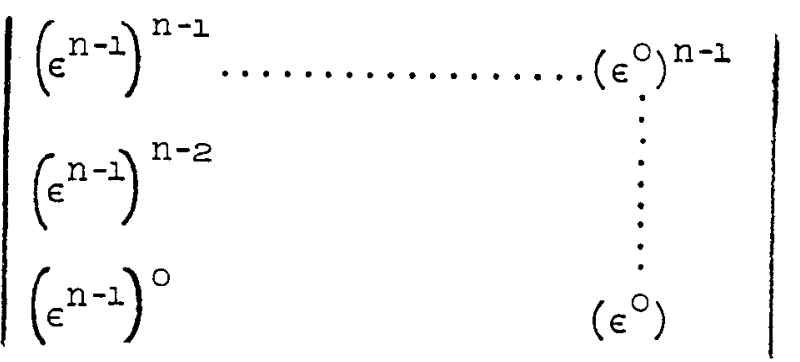

We now use the classical result that

$$
\begin{aligned}
\Delta\left(x_{1}, \ldots, x_{n}\right) & \equiv\left|\begin{array}{ccc}
x_{1}^{n-1} & x_{2}^{n-1} & \cdots \\
x_{1}^{n-2} & x_{2}^{n-2} & x_{n}^{n-1} \\
x_{1}^{0} & \vdots & x_{n}^{0}
\end{array}\right| \\
& =\left(x_{1}-x_{2}\right)\left(x_{1}-x_{3}\right) \cdots\left(x_{1}-x_{n}\right)\left(x_{2}-x_{3}\right) \cdots \\
& =i<j\left(x_{i}-x_{j}\right)
\end{aligned}
$$

Hence, taking into account that $\left(\epsilon^{l_{i}}-\epsilon^{l}{ }^{l}\right) \underset{\Phi \rightarrow 0}{\approx} i \Phi\left(l_{i}-l_{j}\right)$ we obtain

$$
N=\frac{\Delta\left(l_{1}, l_{2}, \cdots \cdot, l_{n}=0\right)}{\Delta(n-1, n-2, \cdots, 0)}
$$

Note that $\Delta(n-1, n-2, . . . .0)=(n-1) !(n-2) ! \ldots . . .1 !$ 
We illustrate, as an example, the calculation of the dimension of the regular representation of $\mathrm{SU}_{6}$ which we know already to be of dimension $6^{2}-1=35$.

\begin{tabular}{|c|c|c|c|}
\hline$f$ & $\ell$ & $n$ & \\
\hline 2 & 7 & 5 & \\
\hline 1 & 5 & 4 & 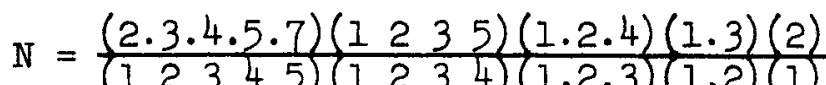 \\
\hline 1 & 4 & 3 & $(12345)(1234)(1.2 .3)(1.2)(1)$ \\
\hline 1 & 3 & 2 & $N=35$ \\
\hline 1 & 2 & 1 & \\
\hline 0 & 0 & 0 & \\
\hline
\end{tabular}

It is sometimes convenient to label differently the representation. Let $\lambda_{1}$ be the number of columns of length one, $\lambda_{2}$ of length two, etc., of a Young tableau. Then

$$
\begin{aligned}
& l_{1}=\lambda_{1}+\lambda_{2}+\cdots+\lambda_{n-1}+n-1 \\
& \ell_{2}=\lambda_{2}+\cdots+\lambda_{n-1}+n-2 \\
& \vdots \\
& l_{n-1}=\lambda_{n-1}+1 \\
& l_{n-1}=0
\end{aligned}
$$

and

$$
\begin{aligned}
& l_{1}-l_{2}=\lambda_{1}+1, l_{1}-l_{3}=\lambda_{1}+\lambda_{2}+2, l_{1}-l_{n}=\lambda_{1}+\ldots+\lambda_{n-1}+n-1 \\
& l_{2}-l_{3}=\lambda_{2}+1, l_{2}-l_{4}=\lambda_{2}+\lambda_{3}+2 \ldots \ldots \ldots
\end{aligned}
$$

Hence

$$
\begin{gathered}
\mathbb{N}=\frac{\left(\lambda_{1}+1\right)\left(\lambda_{2}+1\right) \cdots\left(\lambda_{n-1}+1\right)\left(\lambda_{1}+\lambda_{2}+2\right) \ldots\left(\lambda_{n-2}+\lambda_{n-1}+2\right) \ldots\left(\lambda_{1}+\lambda_{2} \ldots+\lambda_{n-1}+n-1\right)}{1 ! 2 ! \cdots(n-1) !} \\
-18-
\end{gathered}
$$


Finally, if $\mathrm{n}$ is much larger than the number of rows $r$ of a Young tableau, we write

$$
\mathbb{N}=\frac{\Delta\left(\nu_{1}, \nu_{2}, \ldots \nu_{r}\right)}{\nu_{1} ! v_{2} ! \ldots \nu_{r} !} \times \frac{\left(\nu_{1}+n-r\right) !\left(\nu_{2}+n-r\right) ! \ldots\left(\nu_{r}+n-r\right) !}{(n-1) !(n-2) ! \ldots(n-r) !}
$$

where $v_{i}=f_{i}+r-i$ and $i$ runs from $l$ to $r$ only. Asymptotically

$$
N \cong \frac{\Delta\left(v_{1}, v_{2}, \cdots v_{r}\right)}{v_{1} ! v_{2} ! \cdots v_{r} !} n^{f}
$$

which gives a quick estimate of $\mathrm{N}$.

\section{THE GENERATORS OF $\mathrm{SU}_{\mathrm{n}}$}

We have already noted that any $g$ belonging to $\mathrm{SU}_{\mathrm{n}}$ can be written in the form

$$
g=e^{i h}
$$

where $h$ is a rermitian traceless $n \times n$ matrix. It will be convenient, in order to get a parametrization of the group, to choose a basis of $n^{2}-1$ linearly independent such matrices called the generators of the group. For $\mathrm{SU}_{2}$ these are the famous Pauli matrices corresponding to spin. Of fundamental importance are the commutation relations satisfied by the generators; a matrix representation of the generators which satisfies these relations yields a representation of the unitary group.

A convenient choice of basis introduces $n^{2}$ traceless hermitian matrices with one constraint. We define first $\mathrm{n}$ diagonal matrices $\mathrm{H}_{2}, \mathrm{H}_{2} \ldots, \mathrm{H}_{\mathrm{n}}$ such that $H_{i}$ has diagonal elements $-\frac{1}{n}$ except for the $i-t h$ element which 
is equal to $\frac{n-1}{n}$,

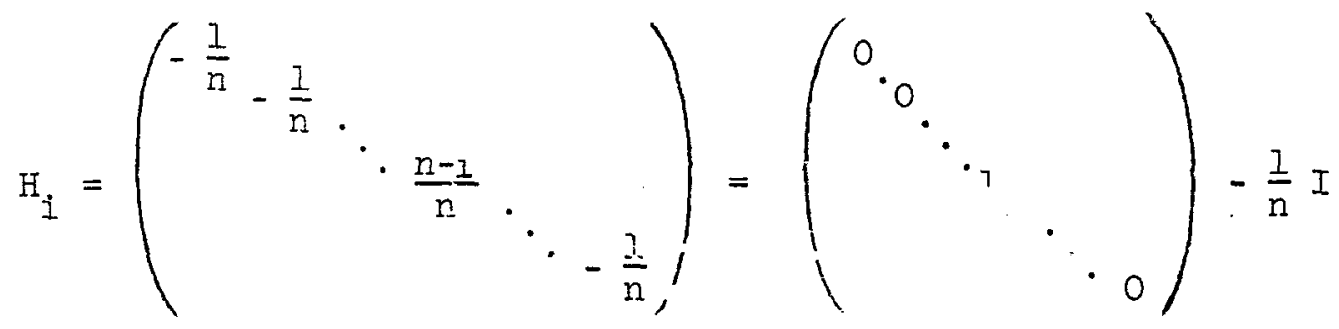

with

$$
\sum_{i=3}^{n} H_{i}=0
$$

Next we introduce matrices $E_{j k}^{(1)}$ and $E_{j k}^{(2)}$ for all $j<k$, which

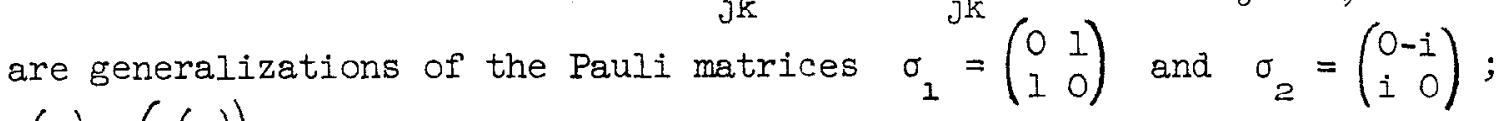
$E_{j k}^{(1)}\left(E_{j k}^{(2)}\right)$ has zero entries except at the intersection of the $j-$ th row and the $k$-th column where it is $I(-i)$, and the $k$-th row and the $j$-th column where it is $I(+i)$

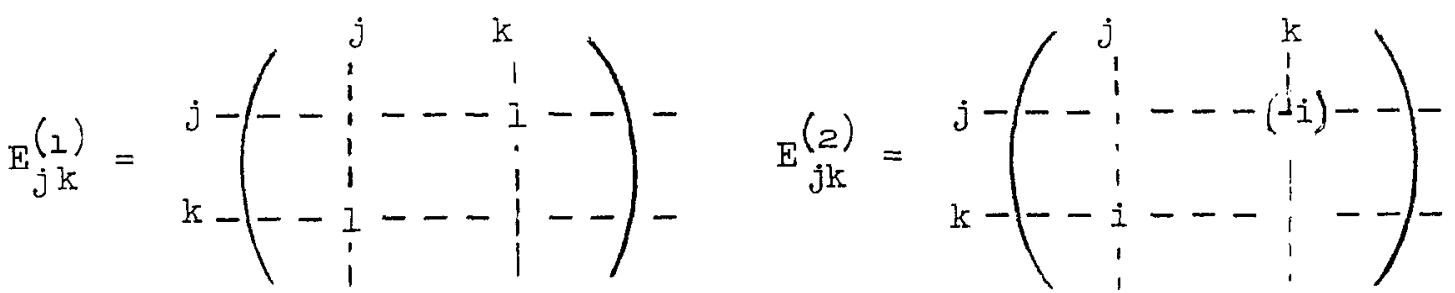

The set of matrices $H_{i}, E_{j k}^{(1)}$ and $E_{j k}^{(2)}$ together with the constraint (3), form a basis in terms of' which we can expand an arbitrary traceless hermitian matrix $h$.

Let us compute the commutation relations of these matrices. For that purpose, we introduce column vectors $e^{(1)} \ldots e^{(n)}$ such that the only nonvanishing component of $e^{(i)}$ is the $i$-th component equal to $I$. 
Then one obtains

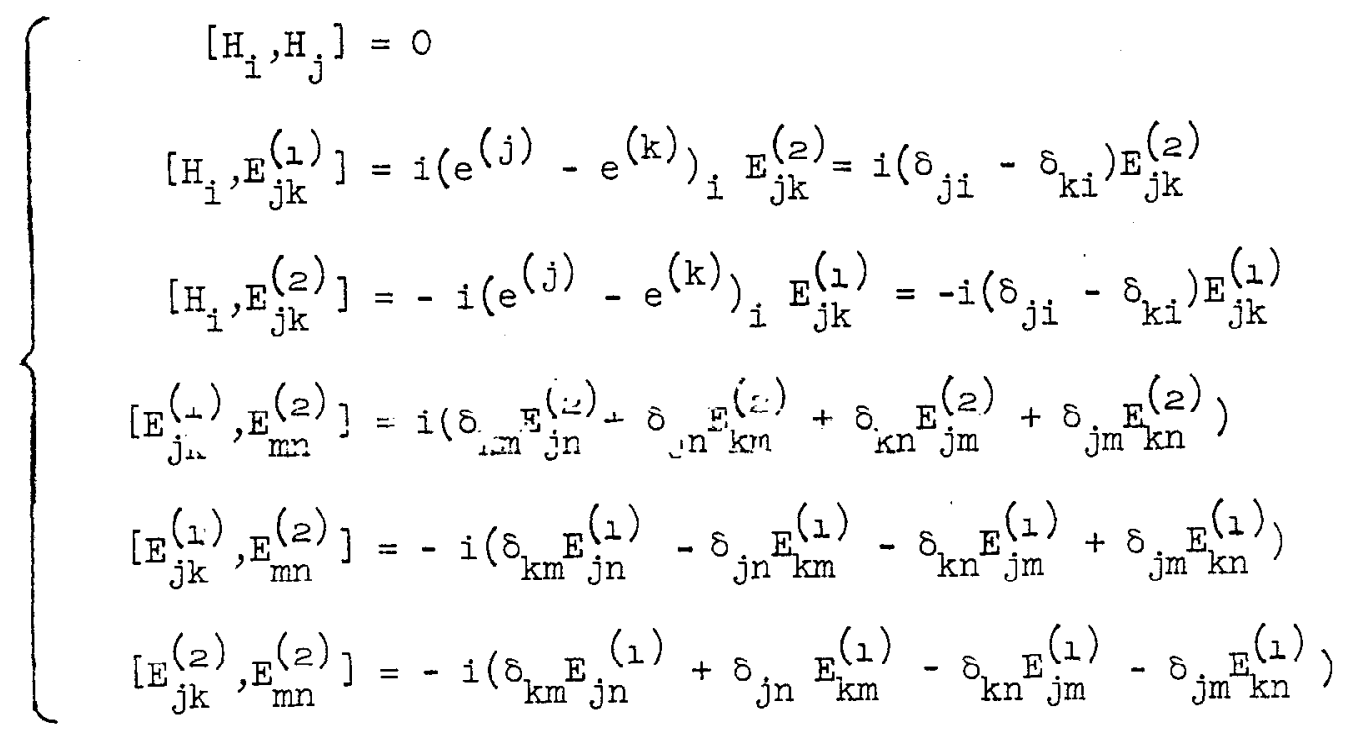

where for convenience of notation heve sat

$$
E_{j j}^{(1)} \equiv 2 H_{j}, E_{j k}^{(1)}=E_{k j}^{(1)} \text { and } E_{j k}^{(2)}=-E_{k j}^{(2)} \text { for } j>k
$$

This set of commutation rules constitutes the "Lie algebra" of the group $\mathrm{SU}_{\mathrm{n}}$. The elements of this algebra are the generators of the group. Actually the relations (5) can be written in a simpler form due to Cartan. Introduce the non-hermitian matrices $E_{j k}$ for $j \neq k$ with zeroes everywhere except at the intersection of the $j$-th and the $k$-th column,

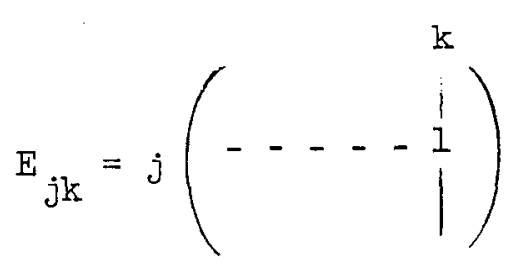

In terms of $\mathrm{E}_{\mathrm{jk}}$, we have

$$
\begin{aligned}
& E_{j k}^{(1)}=E_{j k}+E_{k j} \\
& E_{j k}^{(2)}=-i\left(E_{j k}-E_{k j}\right)
\end{aligned}
$$

Note that $E_{j k}$ is no longer hermitian. 
Then the cormutation relations take the canonical form

$$
\begin{aligned}
& {\left[\mathrm{H}_{i}, \mathrm{H}_{j}\right] }=0 \\
& {\left[\mathrm{H}_{i}, \mathrm{E}_{j \mathrm{k}}\right] }=\left[\mathrm{e}^{(j)}-e^{(\mathrm{k})}\right]_{i} \mathrm{E}_{j \mathrm{k}}=\left(\delta_{i j}-\delta_{i k}\right) \mathrm{E}_{j \mathrm{k}} \\
& {\left[\mathrm{E}_{j \mathrm{~K}}, \mathrm{E}_{m n}\right] }=\mathrm{E}_{j n}{ }^{i} \mathrm{~km} \\
&
\end{aligned}
$$

The vectors $\left[e^{(i)}-e^{(j)}\right]$ are the roots of the algebra. If we denote in $n$ dimensional space the components of a vector by $x_{1}, \cdot \ldots, x_{n}$, the roots are seen to satisfy the equation $x_{z}+x_{2} \cdot \cdot+x_{n}=0$. Hence the roots are $n^{2}-n$ vectors in an $n-1$ dime sional space. For $n=3$ we get the following six roots $\pm\left(e^{(1)}-e^{(2)}\right), \pm\left(e^{(2)}-e^{(3)}\right), \pm\left(e^{(3)}-e^{(1)}\right)$. These are all of length $\sqrt{2}$ and they subtend among themselves angles which are multiples of $\pi / 3$ since the cosine of this angle is \pm 1 or $\pm I / 2$. The overall scale factor is irrelevant. The resulting diagram

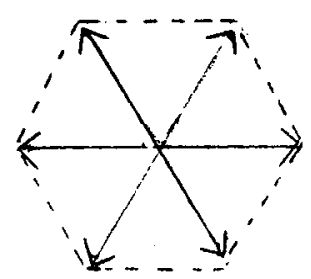

is well known from the eight-fold way of Gell-Mann and Ne'eman. Generally cosines of the angles between roots will take only the values $\pm 1, \pm 1 / 2,0$. This is illustrated in the root diagram for $\mathrm{Su}_{4}$ which is drawn in the 3-dimensional hyperplane $x_{1}+x_{2}+x_{3}+x_{4}=0$. (the roots join the center of a cube to the midpoints of its 12 edges in agreement with the fact that there are $n^{2}-n=(4)^{2}-4=12$ roots). 


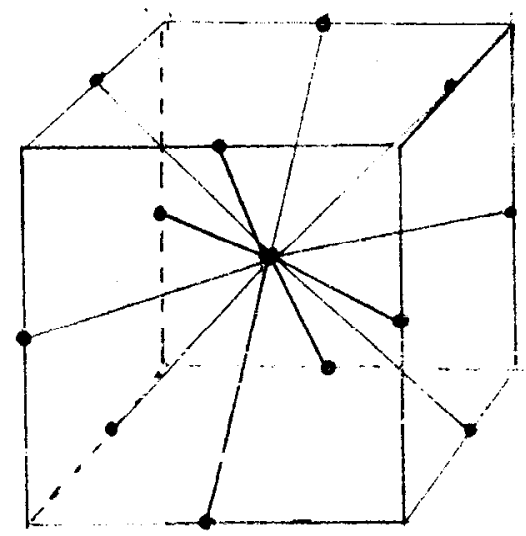

It is straightforward to show that the representations of the unitary group obtained from transformations in the tensor space can also be expressed in terms of generators satisfying the commutation relations (5) and (9). We note simply that the reducible kronecker product is given by

$$
e^{i h} \times e^{i h} \times \cdot \cdot \times e^{i h}=e^{i H}
$$

where

$$
\mathrm{H}=\mathrm{h} \times 1 \times 1 \times \times 1 \oplus 1 \times \mathrm{h} \times 1 \times 1 \times \times 1 \oplus \cdot . \oplus 1 \times 1 \times \ldots \times \mathrm{h}
$$

An important point is that the representations of $\mathrm{H}$ obtained from the irreducible representations of the group are clearly irreducible representations of the lie algebra, and that the converse is true. This is the basis, for example, of the weli-known method in quantum mechanics to obtain the irreducible representation of $\mathrm{SU}_{2}$ by construcing the representations of the spin operators satisfying the "angular momentum commutation relations."

The $\mathrm{H}_{i}$ commute among themselves; hence they can be simultaneously diagonalized. The set of $n$ eigenvalues $H_{i} u=m_{i} u$ (with vanishing sum) is called a weight of the representation. The irreducible representations are uniquely characterized by their highest weight. The adjective highest refers to an 
ordering of the weights in which $\left(m_{1} \ldots m_{n}\right)$ is said to be higher than $\left(m_{1}^{\prime} \ldots m_{n}^{\prime}\right)$ if the first non-vanishing difference $m_{i}-m_{i}^{\prime}$ is greater than zero. To each weight we can associate a vector $m_{1} e_{1}+\ldots+m_{n} e_{n}$ in the n-l dimensional space which already was used for the roots. One can show that in our case, apurt from the condition $\sum_{1}^{n} m_{i}=0$, we must also have

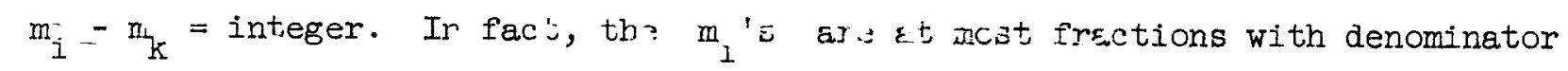
$n$ which differ by integers. The highest welght appears as a linear combination with non-negative integral coefficients of $\mathrm{n}-\mathrm{I}$ fundamental ones*

$$
\begin{aligned}
& M^{(1)}=\left(\frac{n-1}{n}, \frac{-1}{n}, \cdots \cdot \ldots \cdot \ldots \frac{-1}{n}\right) \\
& M^{(2)}=\left(\frac{n-2}{n}, \frac{n-2}{n}, \frac{-2}{n}, \cdots \cdot \cdot \frac{-2}{n}\right) \\
& M^{(3)}=\left(\frac{n-3}{n}, \frac{n-3}{n}, \frac{n-3}{n}, \frac{-3}{n}, \ldots \frac{-3}{n}\right) \\
& M^{(p)}=\left(\frac{n-p}{n}, \cdots \cdots, \frac{-p}{n}, \ldots \frac{-p}{n}\right) \\
& M^{(n-1)}=\left(\frac{1}{n}, \ldots \ldots . . . \frac{1}{n}, \frac{-(n-1)}{n}\right)
\end{aligned}
$$

First we recognize in the weight $\mathrm{M}^{(1)}$ the set of eigenvalues of the operators $\mathrm{H}_{2}, . . \cdot \mathrm{H}_{n}$ corresponding to the eigenvector $\mathrm{y}_{I}=\left(\begin{array}{l}1 \\ 0 \\ 0 \\ 1\end{array}\right)$ in the defining n-dimensional representation of the group. (We define analogously the coordinate vectors $\mathrm{y}_{2} \cdot \cdot \mathrm{y}_{\mathrm{n}} \cdot$ ) It is clearly the highest possible weight and corresponds to the Young tableau $\square$. We shall use the compact notations of exterior calculus to denote antisymmetric tensors. Then consider the second rank antisymmetric tensors and examine the result of $H_{1}$ acting on $\mathrm{y}_{1} \wedge \mathrm{y}_{2}$. If $\mathrm{g}$ acts as

$$
g\left(y_{1} \wedge y_{2}\right)=\left(g y_{1}\right) \wedge\left(g y_{2}\right)
$$

\footnotetext{
${ }^{*}$ See Reference 5 .
} 
then with $g \simeq 1+i \in H \quad(\epsilon$ small $) \quad H$ acts as

$$
\mathrm{H}\left(\mathrm{y}_{1} \Lambda \mathrm{y}_{2}\right)=\left(\mathrm{Hy}_{1}\right) \Lambda \mathrm{y}_{2}+\mathrm{y}_{1} \Lambda \mathrm{H}\left(\mathrm{y}_{2}\right)
$$

Using the explicit form of $H_{i}$ given above, one finds

$$
\begin{aligned}
& \mathrm{H}_{1}\left(\mathrm{y}_{1} \wedge \mathrm{y}_{2}\right)=\frac{\mathrm{n}-1}{-} \mathrm{v}_{1}{\mathrm{y}_{\mathrm{z}}}_{\mathrm{z}}+\left(-\frac{\dot{1}}{\mathrm{n}}\right) \mathrm{r}_{\mathrm{j}} \mathrm{y}_{\mathrm{z}}=\frac{\mathrm{n}-2}{\mathrm{n}} \mathrm{y}_{1} \Lambda_{2} \\
& \mathrm{H}_{2}\left(\mathrm{y}_{1} \Lambda \mathrm{y}_{2}\right)=\frac{-1}{\mathrm{n}} \mathrm{y}_{1} \Lambda \mathrm{y}_{2}+\frac{\mathrm{n}-1}{\mathrm{n}} \mathrm{y}_{1} \Lambda \mathrm{y}_{2}=\frac{\mathrm{n}-2}{\mathrm{n}} \mathrm{y}_{1} \Lambda \mathrm{y}_{2} \\
& \mathrm{H}_{\mathrm{i}}\left(\mathrm{y}_{1} \Lambda \mathrm{y}_{2}\right)=\frac{-1}{\mathrm{n}} \mathrm{y}_{1} \Lambda \mathrm{y}_{2}-\frac{1}{\mathrm{n}} \mathrm{y}_{1} \Lambda \mathrm{y}_{2}=\frac{-2}{\mathrm{n}} \mathrm{y}_{1} \Lambda \mathrm{y}_{2}, \quad \mathrm{i} \geq 2
\end{aligned}
$$

The weight just obtained is in fact the highest weight of the representation. Hence the second weight $M^{(2)}$ corresponds to the representation previously described in terms of antisymmetric second rank tensors or $\square$. There is obviously no difficulty in using the previous technique to prove that ${ }_{M}(p)$ corresponds to the representation in terms of antisymmetric tensors of rank $p: \square p$ boxes.

Accordingly, the $n-1$ representations of $S U_{n}$ obtained in terms of antisymmetric tensors of $\operatorname{rank} 1,2,3, \ldots . n-1$ are the $n-1$ fundamental representations of the group. Once these representations are known it is possible to form direct products of representations (see below) in such a way that at each step one gets only one new representation.

We recall that to each Young tableau (that is, to each representation) we attached two series of $n-1$ numbers, (i) $f_{I}, f_{2} \cdot f_{n-I}\left(f_{n}\right.$ is always identically zero), giving the number of boxes in each row $f_{1} \geq f_{2} \cdot \geq f_{n-1}$, and (ii) $\lambda_{1}, \lambda_{2} \cdot \cdot \lambda_{n-1}, \lambda_{1}$ being the number of 
columns of length one, and so on ..... . This second set is in direct relation to the highest weight of the representation which is equal to $M=\lambda_{1} M^{(1)}+\lambda_{2} M^{(2)}+\cdots+\lambda_{n-1} M^{(n-1)}$.

Before leaving the subject of infinitesimal transformations, it is interesting to notice that among the special unitary groups, $\mathrm{SU}_{2}$ and $\mathrm{SU}_{4}$, turn out to be "isomorphic in the small" to the rotation groups in 3 and 6 dimensions. This means they have the same tie algrora. The first fact is of constant use in the study of the 3-dimensional rotation group. In terms of group theory, the second homomorphism $\mathrm{SU}_{4} \rightarrow \mathrm{R}_{6}$ can be understood as follows: both $\mathrm{SU}_{4}$ and $\mathrm{R}_{6}$ depend on 15 (real) parameters. If we look at the representation of $\mathrm{SU}_{4}$ in terms of antisymmetric tensors of rank two, we find the representation to be of dimeasion 6 , equivalent to its complex conjugate (see above). A little algebra shows that indeed in that case one can find a basis in terms of which the representative matrices are real so that they correspond to rotations. It is then a simple matter to show that one gets all six dimensional rotations in that manner.

More generally, it can be shown that all groups having the same Lie algebra as $\mathrm{SU}_{\mathrm{n}}$ are isomorphic to $\mathrm{SU}_{\mathrm{n}}$ divided by a subgroup of its center. The center of $\mathrm{SU}_{\mathrm{n}}$ is the discrete abelian group which consists of multiples of the identity with determinant one, $u: u_{r}=e^{2 i \pi^{r}} I$ where $r=1,2 \ldots n$.

\section{DECOMPOSITION OF THE PRODUCT OF TWO REPRESENTATIONS OF $\mathrm{SU}_{\mathrm{n}}$}

In many applications one faces the following problem. Let $\vartheta^{(1)}$ and $\eta f(2)$ be the carrier spaces of two irreducible represenations of a group $G$, $A^{(I)}(g)$ and $A^{(2)}(g)$. Then the Kronecker product $V^{(I)} \otimes V^{(2)}$ is the carrier space of the product $A^{(I)}(g) \otimes A^{(2)}(g)$ which is generally a reducible representation of the group. Then the question arises to decompose $A^{(1)} \otimes A^{(2)}$ in its irreducible parts. 
There exist various ways to solve this problem. We will concentrate here on the description of a particularly simple method adapted to the case of $\mathrm{SU}_{\mathrm{n}}$. In this case the carrier spaces $\vartheta^{(1)}$ and $\mathscr{V}^{(2)}$ are composed of tensors with certain symetry properties. Consider a typical elenent of the product

$$
s_{i_{1}} \ldots i_{f_{1}} \quad T_{j_{1}} \ldots j_{f_{2}}
$$

It may be considered as a tensor with $f_{1}+f_{2}$ indices. As such we have a universal procedure to decompose it into parts of maximal symmetry (see Section II). If $S$ and $T$ were not satisfying certain symmctry conditions already, we would thus get each representation with a Youns takieau of $f_{1}+f_{2}$ boxes a certain number of times (in fact, a number of times equal to the dimension of the representation of the symmetric group in $f_{1}+f_{2}$ objects which corresponds also to the same tableau). However, we must take into account the conditions imposed on $\mathrm{S}$ and $\mathrm{T}$. It is clear that the following statement will be true $\because$ in any case. The only representations of $\mathrm{SU}_{\mathrm{n}}$ which appear in the decomposition of the product of two representations corresponding to Young tableaux with $f_{1}$ and $f_{2}$ boxes are those corresponding to tableaux with $f_{1}+f_{2}$, $f_{1}+f_{2}-n, f_{1}+f_{2}-2 n, \ldots$ boxes.

The possibility of subtracting the columns of $n$ boxes explains the statement of the previous proposition. We now give the recipe for solving the decomposition problem. (The reader might find it useful before using the general method to solve the problem for the simple case of the product of an arbitrary representation with $\square$ and then compare.) 
General Recipe

(i) Let
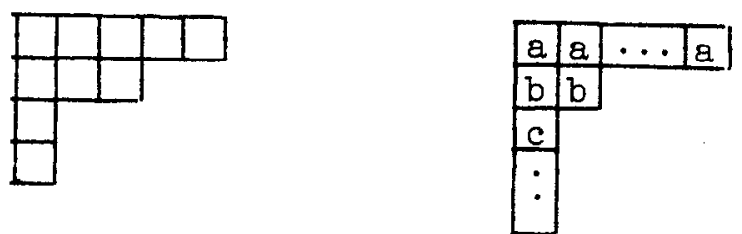

be the two

two representations. Choose one of those as the trunk on which the representations contained in ihe product wiil b: bisiz. Then iabel the boxes in the first row of the second tableau $a$, the boxes in the second line $b$, the boxes in the third line $c$, and so on.

(ii) Add one box labeled a to the first tableau in all possible ways so that it remains a tableau, i.e., the first row of length greater than or equal to the second row, etc. Then cdd a second box labeled a (if any) always requiring that the resultant object be a tableau. When the "a's" are exhausted, use the "b's", then the "c's", and so on.

(iii) In the process described in (ii) never let two boxes with the same label stand in the same column.

(iv) At the end of the process keep only those tableaux with no more than $\mathrm{n}$ rows. (Later on the columns of $\mathrm{n}$ boxes will be dropped; as we have already mentioned, for $\mathrm{SU}_{\mathrm{n}}$ the columns of $\mathrm{n}$ boxes are irrelevant and can be added or omitted without destroying the meaning of the tableau.)

(v) Among the tableau with no more than $\mathrm{n}$ rows, some will be dropped and some others will be kept. In order to decide which are the relevant ones (which correspond to irreducible representations contained in the decomposition of the product), the following device is used. Take some resultant tableau. Reading from right to left and from the upper end to the lower, collect the labels of the boxes. In the process of recollecting, one should always find a number of " $a$ 's" greater or equal to the number of " $b$ 's", 
a number of " $b$ 's" greater or equal to the number of " $c \mathrm{~s}$ ", and so on. Hence, only certain tableaux satisfying the previous criteria survive - they give the desired decomposition.

It is worthwhile to note that at the end some of the tableaux obtained might be identical ( $1 . e$. , the corresponding representation appears several times); hrwever, with attach ad Inbeis sm idsntica. tableaux must differ by the disposition of the letters. For instance,

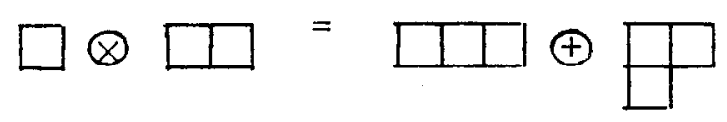

and not

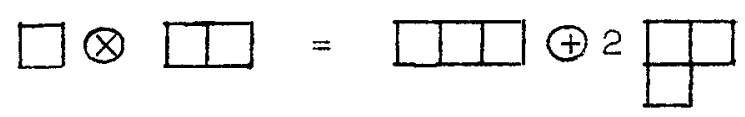

as one could at first have thought. For following the process described above, we label the tableau with two boxes

then attach an "a" to $\square$, thus obtaining

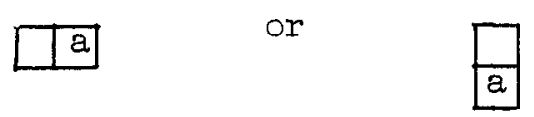

then a second "a"

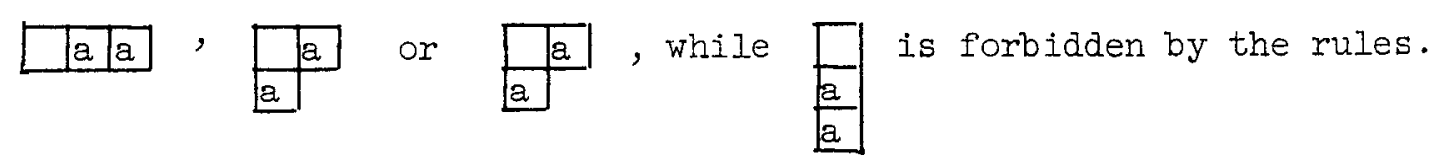

However, the two tableaux $\frac{a}{a}$ differ neither by the tableau nor by the labels and therefore must be treated as a single tableau. This illustrates a second 
point: that a check on the dimensions is generally useful. If $N_{1}$ and $N_{2}$ are the dimensions of $A^{(1)}$ and $A^{(2)}$, and if $N^{(\lambda)}$ denotes the dimension of the irreducible constituents of $A^{(1)} \times A^{(2)}$, we must have

$$
N_{1} \ddot{N}_{2}=\frac{\Gamma N}{(\lambda)}(\lambda)
$$

A last comment before turning to an example - it concerns the case when a diagram contains two rows of the same length, then one must label the two rows differently and proceed as before As an example, consider the problem of decomposing the product of two adjoint represeitations of $\mathrm{SU}_{6}$.

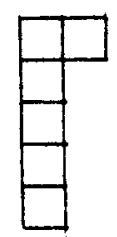

$\otimes$

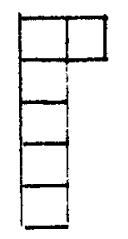

We follow the rules, and label the boxes of one tableau,

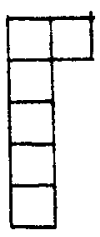

\begin{tabular}{|l|l|}
\hline$a$ & $a$ \\
\hline$b$ & \\
\cline { 1 - 1 } & \\
\cline { 1 - 1 } & \\
\hline$e$ & \\
\hline
\end{tabular}

First Stage
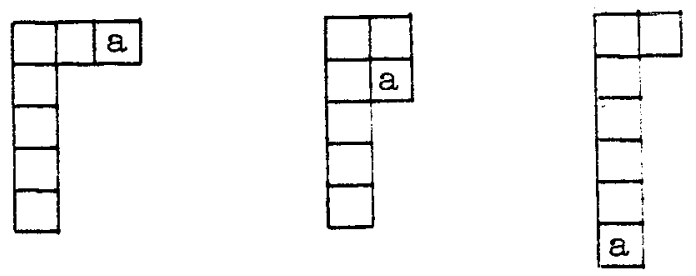
Second Stage
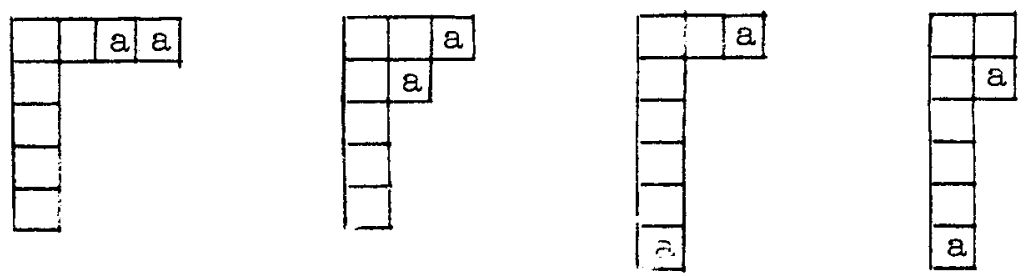

\section{Third Stage}

It is impossible to put a "b" before all "a's" or "c" before "b" (see (v)) and we do not want columns longer than 6 , so the only possibilities are

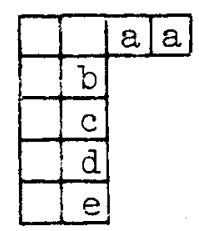

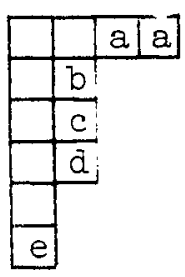
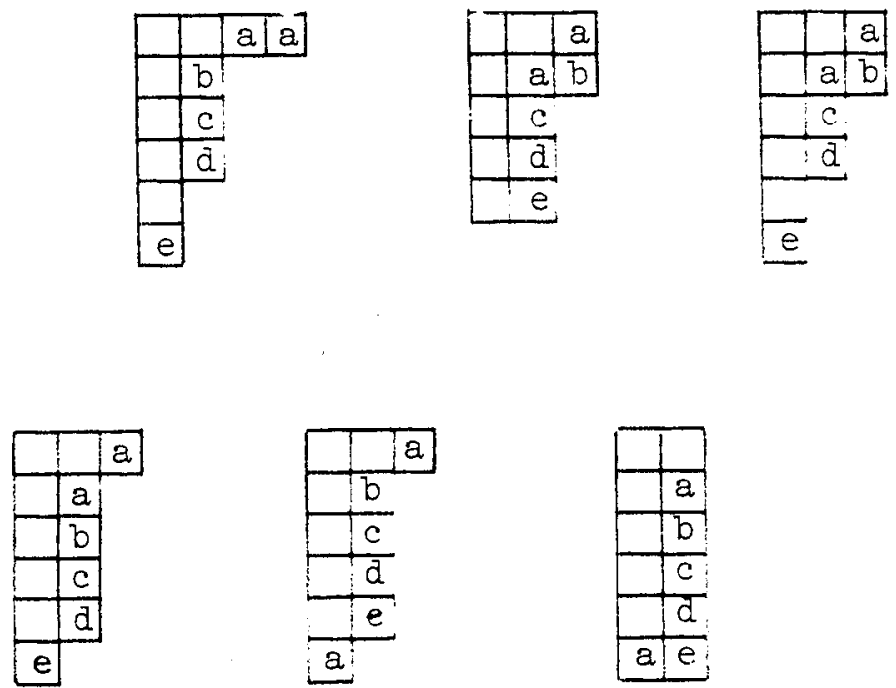

This is the desired decomposition. The final seven representations (with their attached labels) satisfy all the desired criteria. Writing for the symbol of a representation $D^{N}\left(\lambda_{1}, \lambda_{2}, \lambda_{3}, \lambda_{4}, \lambda_{5}\right)$, we have obtained the result:

$$
\begin{aligned}
D^{35}(1,0,0,0,1) \times D^{35}(1,0,0,0,1)= & D^{1}(0,0,0,0,0)+2 D^{35}(1,0,0,0,1) \\
& +D^{189}(0,1,0,1,0)+D^{280}(2,0,0,1,0) \\
& +D^{280}(0,1,0,0,2)+D^{405}(2,0,0,0,2)
\end{aligned}
$$


Indeed, one verifies that

$$
35 \times 35=1+35+35+189+280+\overline{280}+405
$$

The bar recalis the fact that the two representations $(2,0,0,1,0)$ and $(0,1,0,0,2)$ are contragradient t,o sach oiner

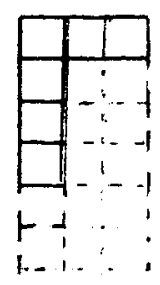

It is clear that in some sense one could have kept the tableaux with more than 6 rows if, instead of dealing with $\mathrm{SU}_{6}$ one were dealing with some $\mathrm{SU}_{\mathrm{n}}, \mathrm{n}>6$. Part of the result previously obtained would still be valid. Hence, it seems desirable to tabulate once and for all the result of the operation relaxing the condition on the number of rows. When applying the result to a given $\mathrm{SU}_{\mathrm{n}}$ one should only keep the tableaux with no more than $n$ rows. This corresponds in fact to solving a related problem for the symmetric group on $f_{1}+f_{2}$ objects: namely, the decomposition of the product of two tensors with given symmetries (described by Young tableaux of $f_{I}$ and $f_{2}$ boxes) into tensors (of rank $f_{1}+f_{2}$ ) of given symmetry (in terms of Young tableaux with $f_{1}+f_{2}$ boxes). This is the so-called decomposition of the "outer product" of two representations of the permutation groups on $f_{1}$ and $f_{2}$ objects. Some tables are given in Section VII. 
VI. THE $\left(\mathrm{SU}_{\mathrm{m}}, \mathrm{SU}_{\mathrm{n}}\right)$ CONTENT OF IRREDUCIBIE REPRESENTATIONS

$$
\text { OF } \mathrm{SU}_{\mathrm{mn}} \mathrm{AND} \quad \mathrm{SU}(\mathrm{m}+\mathrm{n})
$$

The direct product $\left(\mathrm{SU}_{\mathrm{m}}, \mathrm{SU}_{\mathrm{n}}\right.$ ) of two unitary groups $\mathrm{SU}_{\mathrm{m}}$ and $\mathrm{SU}_{\mathrm{n}}$ is a subgroup of $\mathrm{SU}_{\mathrm{mr}}\left(\mathrm{SU}_{\left(\mathrm{i}+\mathrm{r}_{\mathrm{i}}\right)}\right)$. This can be seen by relating ( $\mathrm{SU}_{\mathrm{m}}, \mathrm{SU}_{\mathrm{n}}$ ) to the correspuding linvar trans ormaicn \pm i tne Kronecirer product (direct sum) of two vector spaces of dimension $m$ and $n$. It is clear that an irreducible representation of any group is also a representation, in general reducible, of its subgroups. In this section we consider the problem of finding the irreducible representations of $\left(\mathrm{SU}_{\mathrm{m}}, \mathrm{SU}_{n}\right)$ which are contained in an irreducible representation of $\mathrm{SU}_{\mathrm{mn}}$ of $\mathrm{SU}_{\mathrm{m}+n}$, that is, its $\left(\mathrm{SU}_{\mathrm{m}}, \mathrm{SU}_{n}\right.$ ) content. This has become an important question in applications of groups to the study of elementary particles; for example, we are interested in the $\left(\mathrm{SU}_{2}, \mathrm{SU}_{2}\right)$ or isospin content of $\mathrm{SU}_{3}=\mathrm{SU}_{2+1}$ and in the $\left(\mathrm{SU}_{2}, \mathrm{SU}_{3}\right)$ or spin-unitary spin content of $\mathrm{SU}_{6}=\mathrm{SU}_{2 \times x_{3}}$. We shall discuss these two decompositions separately. To obtain the $\left(\mathrm{SU}_{\mathrm{m}}, \mathrm{SU}_{\mathrm{n}}\right)$ content of an irreducible representation of $\mathrm{SU}_{\mathrm{mn}}$ we consider two vector spaces $V^{(m)}$ and $V^{(n)}$ of dimensions $m$ and $n$ respectively, in which $\mathrm{SU}_{\mathrm{m}}$ and $\mathrm{SU}_{\mathrm{n}}$ operate. The group $\left(\mathrm{SU}_{\mathrm{m}}, \mathrm{SU}_{\mathrm{n}}\right.$ ) corresponds to unitary transformations in the tensor product space $v^{(m)} \times V^{(n)}$, with the scalar product defined by

$$
\left(w^{(m)} \times w^{(n)}, v^{(m)} \times v^{(n)}\right)=\left(w^{(m)}, v^{(m)}\right)\left(w^{(n)}, v^{(n)}\right),
$$

which leave $\left(w^{m}, v^{m}\right)$ and $\left(w^{n}, v^{n}\right)$ invariant, where $w^{(i)}$ and $v^{(i)}$ are vectors in $v^{(i)}, i=m, n$, and $\left(w^{(i)}, v^{(i)}\right)$ is the scalar product of ${ }_{W}(i)$ and $v^{(i)}$. It is then clear that $\left(S_{m}, S U_{n}\right)$ is a subgroup of $S_{m n}$ which operates in $\mathrm{V}^{(\mathrm{m})} \times \mathrm{V}^{(\mathrm{n})}$. 
The components of a vector in $\mathrm{V}^{(\mathrm{m})} \times \mathrm{V}^{(\mathrm{n})}$ can be written in the form $V_{(i, \alpha)}$, where $i$ runs from $I$ to $m$ and $\alpha$ from $l$ to $n$. Hence a tensor of rank $f$ has the form

$$
T_{\left(i_{1} \alpha_{1}\right),\left(i_{2} \alpha_{2}\right), \ldots\left(i_{f} \alpha_{f}\right)}
$$

To obtain the carrier space of an irredusible representation of $\mathrm{SU}_{\mathrm{m}}$ we have to impose a "maxima symuetry conuition" $\omega_{1}$ : the indices of $T$ (see Sec.III). In applying this symmetry condition to $T$ we have to permute pairs of indices $\left(i_{j}, \alpha_{j}\right)$ at the same time. On the other hand, the carrier space for the irreducible representations of $\left(\mathrm{SU}_{m}, \mathrm{SU}_{n}\right)$ is obtained by imposing a maximal symmetry condition on the indices $i$ and $\alpha$ separatoly. Hence to get the $\left(\mathrm{SU}_{m}, \mathrm{SU}_{\mathrm{n}}\right)$ content of an irreducible representation of $\mathrm{SU}_{\mathrm{mn}}$ we have to decompose the tensors which satisfy symetry conditions with respect to the permutation of pairs of indices $(i, \alpha)$ into the sum of tensor which satisfy such conditions for separate permutation of the indices $i$ and $\alpha$.

Consider as an example the representation of $\mathrm{SU}_{\mathrm{mn}}$, corresponding to the Young tableau:

It is described in terms of tensors

$$
T_{i_{1} \alpha_{1}, i_{2} \alpha_{2}}=T_{i_{2} \alpha_{2}, i_{1} \alpha_{1}}
$$

One can obviously write

$$
\begin{aligned}
T_{i_{1} \alpha_{1}, i_{2} \alpha_{2}} & =\frac{1}{2}\left(T_{i_{1} \alpha_{1}, i_{2} \alpha_{2}}+T_{i_{2} \alpha_{1}, i_{1} \alpha_{2}}\right) \\
& +\frac{1}{2}\left(T_{i_{1} \alpha_{1}, i_{2} \alpha_{2}}-T_{i_{2} \alpha_{1}, i_{1} \alpha_{2}}\right)
\end{aligned}
$$


The first parenthesis is symmetric in the interchange of $i_{1}$ and $i_{2}$; it is also invariant with respect to the interchange of $\alpha_{1}, \alpha_{2}$. The second is antisymmetric in the interchange of $i_{2}, i_{2}$, and separately in the interchange of $\alpha_{1}, \alpha_{2}$. The symbolic notation for the decomposition is

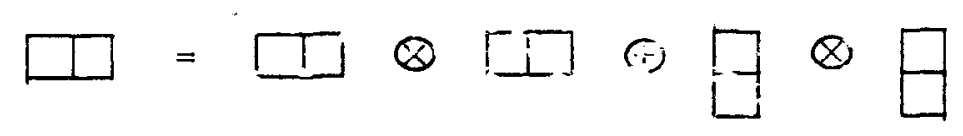

We can make a check on the dimensions. On the left we have a representation of $s J_{m n}$ (and hence of $\left(\mathrm{SU}_{\mathrm{m}}, \mathrm{SU}_{\mathrm{n}}\right)$ ) of dimension

$$
\left(\begin{array}{c}
m n+1 \\
2
\end{array}\right)=\frac{(m n+1) m}{2}
$$

On the right we have a representation of dimension

$$
\left(\begin{array}{c}
m+1 \\
2
\end{array}\right)\left(\begin{array}{c}
n+1 \\
2
\end{array}\right)=\frac{(m+1) m}{2} \cdot \frac{(n+1) n}{2}
$$

and another one of dimension

$$
\left(\begin{array}{l}
m \\
2
\end{array}\right)\left(\begin{array}{l}
n \\
2
\end{array}\right)=\frac{m(m-1)}{2} \cdot \frac{n(n-1)}{2}
$$

Hence we should have

$$
\left(\begin{array}{c}
m+1 \\
2
\end{array}\right)=\left(\begin{array}{c}
m+1 \\
2
\end{array}\right)\left(\begin{array}{l}
n+1 \\
2
\end{array}\right)+\left(\begin{array}{l}
m \\
2
\end{array}\right)\left(\begin{array}{l}
n \\
2
\end{array}\right)
$$

which is indeed satisfied. Now it is apparent that the following general statement is true: "Given an irreducible representation of $\mathrm{SU}_{\mathrm{mn}}$ whose 
Young tableau contains $f$ boxes, the only irreducible representations of $\left(\mathrm{SU}_{m}, \mathrm{SU}_{n}\right)$ it contains are those with $f-\lambda_{m}$ and $f-\lambda^{\prime} n$ boxes respectively, where $\lambda$ and $\lambda^{\prime}$ are integers." Again this stems from the irrelevance of columns with $p$ boxes added to the Young tableaux of an irreducible representative of $\mathrm{SU}_{\mathrm{p}}$.

The decomposition of teisnrs of hikh rank involves a considerable amount of labor. If we are manly interestec in isuiting tise ( $\left.\mathrm{SU}_{\mathrm{m}}, \mathrm{SU}_{\mathrm{n}}\right)$ content of $\mathrm{SU}_{\mathrm{mn}}$ we can use a simpler method based on the observation that this decomposition is directly related to the reduction of the Kronecker product of two representations of the symmetric group $\sum f^{\cdot}$. We note that for fixed values of the indices $i$ and $\alpha$, the telsscrs $T_{\left(i_{1} \alpha_{1}\right),\left(i_{2} \alpha_{2}\right)} \cdots$ with maximal symmetry conditions for permutations on $i$ and $\alpha$ separately, are also basis for the Kronecker product of two representations of $\sum_{f}$. The decomposition of these tensors into tensors with maximal symmetry conditions under simultaneous permutations of $i$ and $\alpha$ leads to the reduction of the corresponding Kronecker product into irreducible parts according to $\sum f^{\circ}$ This leads to the following procedure:

Suppose we want to know whether a given representation $\left(D_{m} \times D_{n}\right)$ is contained in a given representation $D_{m n}$ of $S_{m n}$ symbolized by a Young tableau with $f$ boxes. First, as explained above, one can add to the Young tableau of $D_{m}\left(D_{n}\right)$ a certain number ${ }^{*}$ of columns of length $m(n)$ on the left in order to bring them to a form where it contains $f$ boxes. The Young tableaux obtained in that fashion describe also two irreducible representations of the symmetric group $\sum f^{*}$ Then the given representation $D_{m n}$ of $S_{m n}$ contains the representation $\left(D_{m}, D_{n}\right)$ of $\left(S_{m}, S U_{n}\right)$ as many times as the corresponding representation of $\sum f$ appears in the decomposition of the product This number can of course be zero. 
of the representations of $\sum f$ corresponding to the Young tableaux of $D_{m}$ and $D_{n}$. In other words, what one has to do is to obtain the Clebsch-Gordan series of the corresponding representations of $\sum_{f}$. An important advantage of this method is that it allows us to forget essentially the subscripts $m$ and $\mathrm{n}$. The tabulation of the Clebsch-Gordan series can in fact be made only

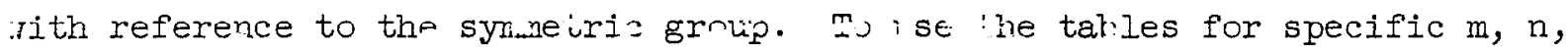
one only has to disregard Young tableaux of more than $m(n)$ rows, and columns of length $m(n)$ (see Section VII).

We now turn our attention to the problem of finding the $\left(\mathrm{SU}_{\mathrm{m}}, \mathrm{SU}_{\mathrm{n}}\right)$ content of a representation of $S_{m+n}$. For this purpose we form the vector space sum $\mathrm{V}^{(\mathrm{m})}+\mathrm{V}^{(\mathrm{n})}$ in which the scalar priauct is now defined by

$$
\left(w^{(m)}+w^{(n)}, v^{(m)}+v^{(n)}\right)=\left(w^{(m)}, v^{(m)}\right)+\left(w^{(n)}, v^{(n)}\right)
$$

The transformations $\left(\mathrm{SU}_{\mathrm{m}}, \mathrm{SU}_{\mathrm{n}}\right.$ ) in this space form a subgroup of $\mathrm{SU}_{\mathrm{m}+\mathrm{n}}$ which leaves $\left(\mathrm{w}(\mathrm{m}), \mathrm{v}^{(\mathrm{m})}\right)$, and $\left(\mathrm{w}^{(\mathrm{n})}, \mathrm{v}^{(\mathrm{n})}\right)$ separately invariant. The components of a vector in $V^{(m)}+V^{(n)}$ are now written in the form $V_{i}$, where $i$ runs from 1 to $m+n$ with the convention that for $i=1 \ldots m(i=m+1 \ldots m+n)$ these components belong to $v^{m}\left(v^{n}\right)$. Then for a tensor of rank $f$ we write $\mathrm{T}_{I_{1}} \ldots i_{f}$. Now if we want to build irreducible representations of (SU $\mathrm{m}_{\mathrm{n}}, \mathrm{SU}_{\mathrm{n}}$ ) we need to consider only tensors in which the index $i_{j}$ runs either from 1 to $m$ or from $m+I$ to $m+n$ and impose maximal symmetry conditions among indices of the same kind. These symmetrized tensors can also serve to induce a representation of $\mathrm{SU}_{\mathrm{m}+\mathrm{n}}$ if we adopt the convention that they have zero components for the absent values of the indices. The representation obtained in this way is reducible and corresponds to the Kronecker product of the two representations of $S_{m+n}$ labeled by the two Young tableaux which previously 
referred to $\mathrm{SU}_{\mathrm{m}}$ and $\mathrm{SU}_{\mathrm{n}}$. The decomposition problem has been solved in Section $V$. This is the basis for the method of obtaining the ( $\left.\mathrm{SU}_{\mathrm{m}}, \mathrm{SU}_{n}\right)$ content of $\mathrm{SU}_{\mathrm{m}+\mathrm{n}}$ which we now describe.

Given a representation of $\left(\mathrm{SU}_{m}, \mathrm{SU}_{n}\right)$ we can associate with it two Young tableaux, one for $S_{m}$ and one for $S U_{p}$, for instance

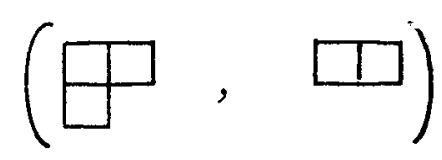

Then we know from Section $V$ how to decompose the "outer" product of the corresponding representations of the symmetric groups, namely

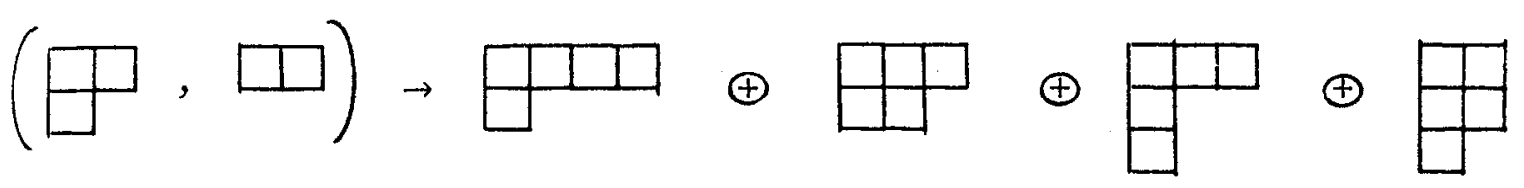

We refer the tableaux of the right-hand side to representations of $\mathrm{SU} \mathrm{m}_{\mathrm{n}}$. Then the given representation of $\left(\mathrm{SU}_{\mathrm{m}}, \mathrm{SU}_{\mathrm{n}}\right)$ appears in the decomposition of the representations of $\mathrm{SU}_{\mathrm{m}+\mathrm{n}}$ which appear on the right-hand side as many times as their multiplicity indicate (in our example 0 or 1 ).

Note that to the Young tableaux for the given representation of $\left(\mathrm{SU}_{\mathrm{m}}, \mathrm{SU}_{\mathrm{n}}\right)$ we can add $\alpha$ columns of length $m$, and $\beta$ columns of length $n$, respectively. In our example we have

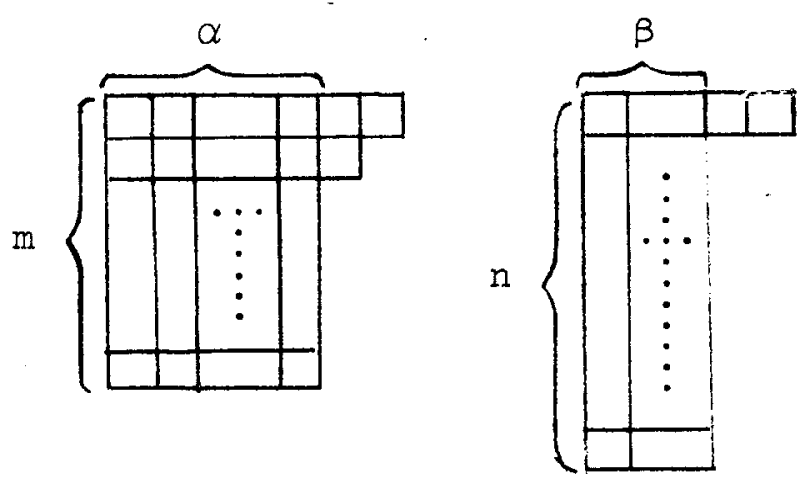


Hence this representation of $\left(\mathrm{SU}_{m}, \mathrm{SU}_{n}\right)$ will also appear in the decomposition of representations of $\mathrm{SU}_{\mathrm{m}+\mathrm{n}}$ with $\alpha \mathrm{m}+\beta_{n}$ extra boxes. In general a given representation of $S_{m+n}$ with a Young tableau of $f$ boxes contains only those representations of $\left(\mathrm{SU}_{\mathrm{m}}, \mathrm{SU}_{\mathrm{n}}\right)$ for which the number of boxes of the two Young tableaux, call them $f_{1}$ and $f_{2}$, are such that

$$
f_{1}+f_{2}+\alpha m+\beta n=f
$$

A special case of this decomposition is Weyl's branching law which gives the $\mathrm{SU}_{\mathrm{n}-1}$ content of a representation of $\mathrm{SU}_{\mathrm{n}}$. Indeed, it corresponds to the $\left(\mathrm{SU}_{\mathrm{n}-1}, \mathrm{SU}_{1}\right)$ content of $\mathrm{SU}_{\mathrm{n}}$ where $\mathrm{SU}_{1}$ is a trivial group reduced to one element. Its representations are all the unity matrix, but may be represented by arbitrary Young tableaux with one row. Suppose we are given a representation $\left(f_{1}^{t}, f_{2}^{\prime}, \ldots, f_{n-2}^{\prime}\right)$ of $S_{n-1}$. First we allow for an arbitrary number of extra columns of length $n-1$ by writing it

$$
\left(f_{1}^{\prime}+\alpha, f_{2}^{\prime}+\alpha, \ldots f_{n-2}^{\prime}+\alpha, \alpha\right)
$$

Then consider a "representation" of $\mathrm{SU}_{1}(\beta)$ and decompose the outer product

$$
\left(f_{1}^{\prime}+\alpha, f_{2}^{\prime}+\alpha, \cdots f_{n-2}^{\prime}+\alpha, \alpha\right)
$$

according to the rules of Section $V, \alpha$ and $\beta$ are chosen in order to find $\left(f_{1}, \ldots, f_{n-1}\right)$ in the decomposition. Clearly a necessary condition is that

$$
f_{1}^{\prime}+\ldots+f_{n-2}^{\prime}+\alpha(n-1)+\beta=f_{1}+f_{2}+\ldots+f_{n-1} .
$$

Then we shall have in the process of decomposition tableaux with rows of length

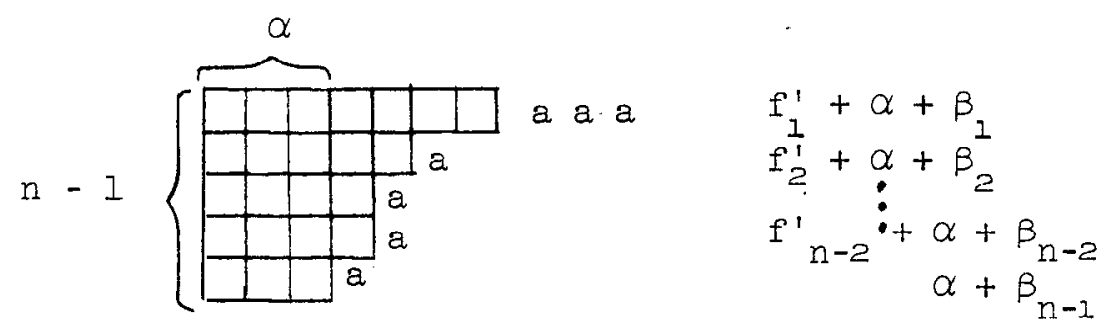


with $\beta_{I}+\ldots+\beta_{n-1}=\beta$ and due to the process of construction ("two a's camnot be in the same column")

$$
\begin{aligned}
& f_{2}^{\prime}+\alpha+\beta_{2} \leq f_{1}^{\prime}+\alpha \\
& f_{3}^{\prime}+c+\beta_{3} \leq f_{2}^{\prime}+\alpha \\
& \alpha+\beta_{n-1} \leq f_{n-2}^{\prime}+\alpha
\end{aligned}
$$

We also want

$$
\begin{aligned}
& f_{1}^{\prime}+\alpha+\beta_{1}=f_{1}^{\prime} \\
& f_{2}^{\prime}+\alpha+\beta_{2}=f_{2}^{\prime} \\
& f_{n-2}^{\prime}+\alpha+\beta_{n-2}=f_{n-2} \\
& \alpha+\beta_{n-1}=f_{n-1}
\end{aligned}
$$

So

$$
\begin{aligned}
\alpha+f_{1}^{\prime} & =f_{1}-\beta_{1} \leq_{1} \\
f_{2} & =f_{2}^{\prime}+\alpha+\beta_{2} \leq f_{1}^{\prime}+\alpha
\end{aligned}
$$

and analogous inequalities for $f_{3}^{\prime}, f_{4}^{\prime} \ldots .$. . The result reads:

$$
\begin{aligned}
& f_{2} \leq \alpha+f_{1}^{\prime} \leq f_{1}--- \\
& f_{\ell+1} \leq \alpha+f_{\ell}^{\prime} \leq f_{\ell} \\
&---- \\
& 0 \equiv f_{n} \leq \alpha \leq f_{n-1} .
\end{aligned}
$$

This set of inequalities is the content of Weyl's branching law: the representations $\left(f_{2}^{\prime} \ldots, f_{n-2}^{\prime}\right)$ of $S_{n-1}$ contained in a representation $\left(f_{1} \ldots f_{n-1}\right)$ of $\mathrm{SU}_{\mathrm{n}}$ are those for which there exists a positive integer (or zero) $\alpha$ 
such that the previous inequalities are satisfied. It appears as a special case of the general method outlined above.

In practice it is better to tabulate the decomposition of "outer" products of representations of the symmetric groups. We are thus able to use these tables to solve two different problems pertaining to the unitary groups. The detai's are diroussad ir the next recrion.

\section{TABLES}

We give below tables which are useful for the various decomposition problems of both the symmetric group $\sum f$ and the unitary group $\mathrm{SU}_{\mathrm{n}}$ (see Sections $V$ and VI). We shall rext, discus: their use.

A. Dimension of representations.

From $\mathrm{SU}_{3}$ to $\mathrm{SU}_{12}$ we give the value of the dimension of the representations up to Young tableaux with 8 boxes. The first column gives the dimension of the corresponding representation for the symmetric group $\sum_{f}$.

B. Decomposition of the "outer product" of two representations of

$$
\begin{aligned}
& \sum f_{1} \text { and } \sum_{f_{2}} \text { with respect to } \sum_{\text {for the product of two representations of } f_{1}+f_{2} \text { and Clebsch-Gordan series }} \text {. }
\end{aligned}
$$

The tables first refer to the decomposition of the "outer product" of two representations of $\sum f_{1}$ and $\sum f_{2}$ or, what is equivalent, to the decomposition of the product of two tensors with given "maximal" symmetries in the $f_{1}$ and $f_{2}$ indices respectively, into teñsors with "maximal" symmetries in the $f_{1}+f_{2}$ indices. In other words, they also solve the problem of decomposing the product of two representations of $\mathrm{SU}_{\mathrm{n}}$ (see Section $\mathrm{V}$ ). As an example, the following representations of $\sum_{2}$ and $\sum_{5}$ induce the 
following representations of $\sum_{7}$ as read in the tables

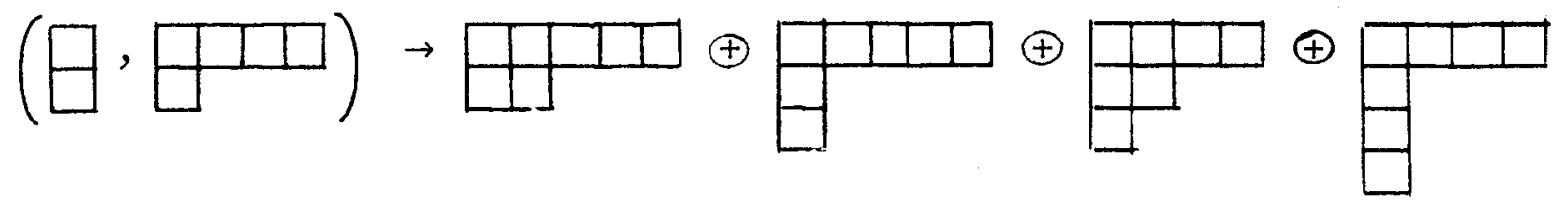

Notice that reading from Table A the dimensions of the corresponding representations of the symmetric groups, one finds,

$$
1 \times 4 \rightarrow 14+15+35+20=84
$$

The dimensions on both sides are not equal, but, the left-hand side always divides the right-hand side.*

When labeling the representations we have used $f_{1} \geq f_{2} \geq \cdots \geq f_{n-1}$; " $f$ 's" equal to zero ere omitted and when

$$
f_{p+1}=\ldots=f_{p+r}=s
$$

instead of repeating "s" $r$ times, we have written $s$.

In using the tables to decompose the Kronecker product of two representations of $\mathrm{SU}_{\mathrm{n}}$, it is necessary

(i) to ignore Young tableaux with more than $n$ rows

(ii) to consider as equivalent two Young tableaux when they differ only in the fact that one has extra columns of $n$ boxes.

*This is due to the following fact. The representation of $\left(\sum f_{1}, \sum_{2}\right)$ of dimension $n_{1} n_{2}$ induces a representation of $\sum_{f_{1}+f_{2}}$ of dimension equal

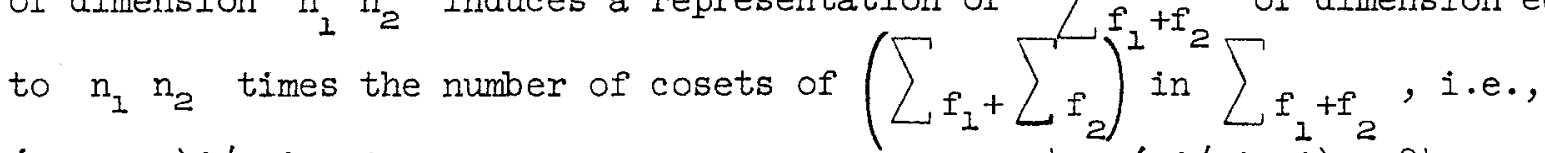
$\left(f_{1}+f_{2}\right) ! / f_{1} ! f_{2} !$. In our example this is $1 \times 4 \times(7 ! / 2 ! 5 !)=84$. 
Example: Using the tables for $\mathrm{SU}_{3}$ one gets

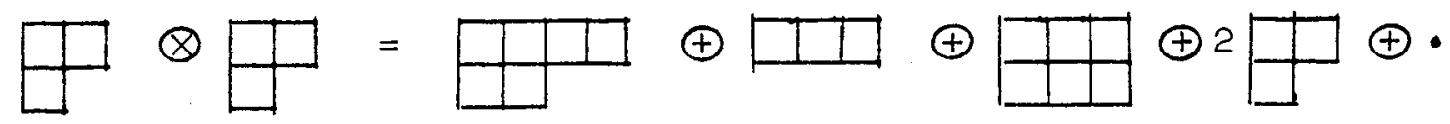

(The dot indicates the one-dimensional representation.) This also reads in familiar language

$(8) \times(8)=(27)+(10)+(\overline{10})+2(8)+(1)$

The dimensions are equal on both sides.

If we turn to. $\mathrm{SU}_{6}$ the same decomposition problem now leads to
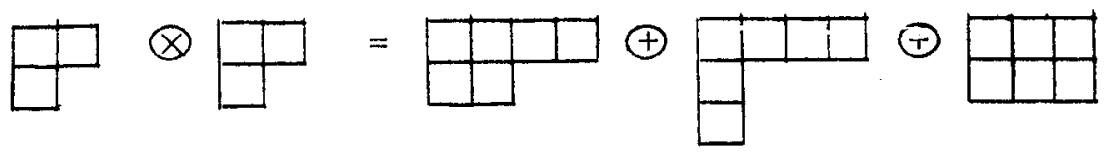

(
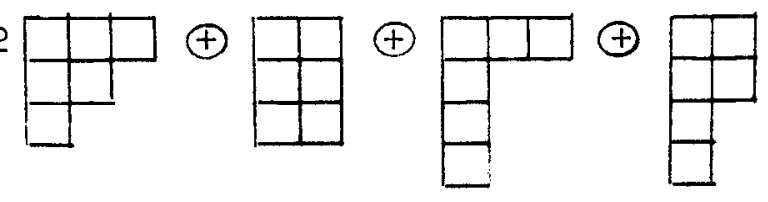

or in terms of the dimensions

$(70) \times(70)=(1134)+(840)+(490)+2(896)+(175)+(280)+(189)$

Finally, the tables are also used "vertically" to find the $\left(\mathrm{SU}_{\mathrm{m}}, \mathrm{SU}_{\mathrm{n}}\right)$ content of an irreducible representation $\mathrm{SU}_{\mathrm{m}+\mathrm{n}}$ (Section VI). In order to do this, select the column of the given representation of $\mathrm{SU}_{\mathrm{m}+\mathrm{n}}$ corresponding to a Young tableau with $f$ boxes in the table $f_{1}+f_{2}=f$.

Each entry in the column is equal to the number of times the representation of $\left(\mathrm{SU}_{\mathrm{m}}, \mathrm{SU}_{\mathrm{n}}\right)$ appearing on the left occurs in the given representation of $\mathrm{SU}_{\mathrm{m}+\mathrm{n}}$. The two partitions corresponding to the Young tableaux appearing on the side of the table correspond to representations of $\left(\mathrm{SU}_{m}, \mathrm{SU}_{n}\right)$ in two ways: (i) the 
first partition refers to $\mathrm{SU}_{\mathrm{m}}$ and the second to $\mathrm{SU}_{\mathrm{n}}$, and, (ii) the first partition refers to $\mathrm{SU}_{\mathrm{n}}$ and the second partition refers to $\mathrm{SU}_{\mathrm{m}}$ " $^{*}$ The only exception to this rule is when the two partitions are identical in which case one reads them only once. The table for $f_{1}+f_{2}=f$ must be trivially completed by extra rows ccrrespondirg to $0+i=f$, that is, the outer products of the representations of $\sum f$ by those of a Eroup.reauced to the identity thus inducing the same representation of $\sum f^{* *}$ As customary we disregard tableaux of more than $m(n)$ rows for $\mathrm{SU}_{m}\left(\mathrm{SU}_{n}\right)$ and columns of length $m(n)$. As an example, consider the representation $\square \square$ of $\mathrm{SU}_{6}$ and let us find its $\left(\mathrm{SU}_{2}, \mathrm{SU}_{4}\right)$ content. Using the tinle for $f_{1}+f_{2}=3$ we find ${ }^{* * *}$

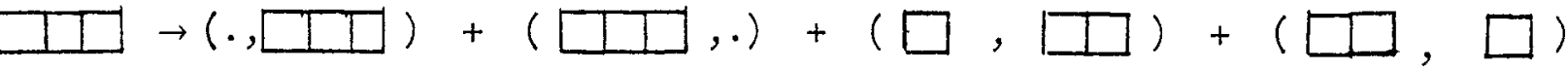
$\left(\mathrm{SU}_{6}\right) \rightarrow\left(\mathrm{SU}_{2}, \mathrm{SU}_{4}\right)$

or

$$
(56)=(1,20)+(4,1)+(2,10)+(3,4)
$$

\footnotetext{
Even if $m=n$.
}

** In the preceding problem of Clebsch-Gordan series for group SU this corresponds to the trivial decomposition of the product of an identity representation $\left(f_{1}^{\prime}=\ldots=f_{n-1}^{\prime}=0\right)$ by a representation $\left(f_{1}, \ldots, f_{n-1}\right)$ thus re$\underset{* * *}{\operatorname{aucing}}$ to $\left(f_{1}, \ldots, f_{n-1}\right) \times(0, \ldots, 0)=\left(f_{1}, \ldots, f_{n-1}\right)$.

Note that the two first terms correspond precisely to the extra rows to be added to the tables. 
C. These give the Clebsch-Gordan series for the symmetric group, and the content of an irreducible representation of $\mathrm{SU}_{\mathrm{mn}}$ in terms of its $\left(\mathrm{SU}_{\mathrm{m}}: \mathrm{SU}_{\mathrm{n}}\right)$ subgroup (Section VI) up to Young tableaus with eight boxes. Reading "horizontally" one finds the Clebsch-Gordan series of the product of two represerivations of $\zeta^{\cdot}$. ixaiple inr $\sum_{4}$ :

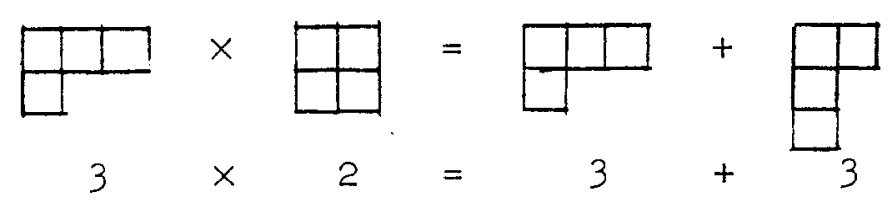

One can also read them "vertically" for a representation of $\mathrm{SU}_{\mathrm{mn}}$, thus collecting on the sides of the table its content in terms of $\left(\mathrm{SU}_{\mathrm{m}}, \mathrm{SU}_{\mathrm{n}}\right)$. To do this, first select in the upper part of the table the Young tableau of a given representation of $\mathrm{SU}_{\mathrm{mn}}$. This same tableau reappears in the lower part of the table. Each entry is the number of times the representations of ( $\mathrm{SU}_{n}, \mathrm{SU}_{\mathrm{m}}$ ) appearing on the left (right) of a corresponding row occurs in the representation of $\mathrm{SU}_{\mathrm{nm}}$ indicated in the top (bottom) of the column. The two partitions corresponding to the Young tableaux appearing on each side of the table, correspond to representations of $\left(\mathrm{SU}_{m}, \mathrm{SU}_{n}\right)$ in two ways: (i) the first partition refers to $\mathrm{SU}_{\mathrm{m}}$ and the second to $\mathrm{SU}_{\mathrm{n}}$; (ii) the first partition refers to $\mathrm{SU}_{\mathrm{n}}$, the second to $\mathrm{SU}_{\mathrm{m}}{ }^{*}$ However, as usual, Young tableaux with more than $m(n)$ rows referring to $\mathrm{SU}_{\mathrm{m}}\left(\mathrm{SU}_{\mathrm{n}}\right)$ are disregarded as well as columns of' length $m(n)$.

Example for $\mathrm{SU}_{6}$ : Reading the table one obtains the $\left(\mathrm{SU}_{2}, \mathrm{SU}_{3}\right)$ content of the following representation (interesting in the case of baryon number

* Except in the case when the two partitions are identical 
two states),

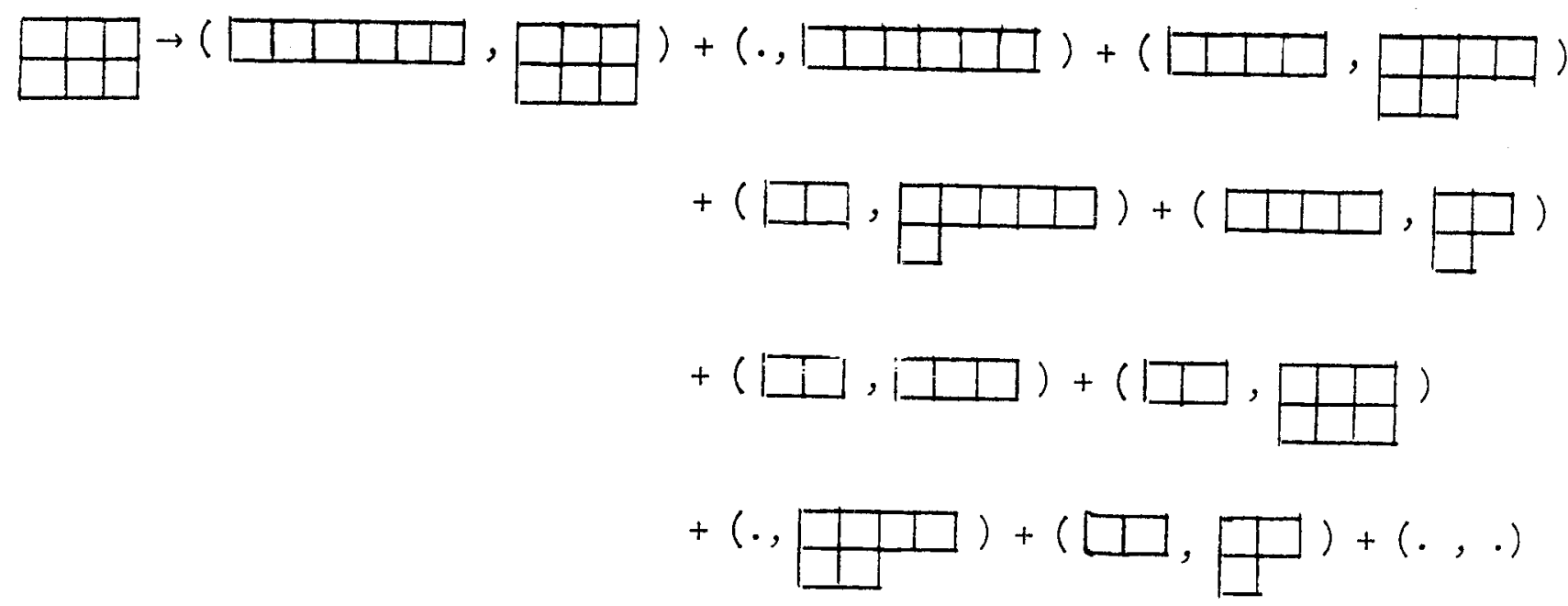

$\left(\mathrm{SU}_{6}\right) \rightarrow\left(\mathrm{SU}_{2}, \mathrm{SU}_{3}\right)$

or, in terms of dimensions

$(490) \rightarrow(7, \overline{10})+(1,28)+(5,27)+(3,35)+(5,8)+(3,10)+(3, \overline{10})+(1,27)+(3,8)+(1,1)$

Of course the sum of the dimensions on the right adds up to the dimension on the left.

In general, when the two representations have the same Young tableau they should not be duplicated (as explained above). To illustrate this remark, considerfor instance the $\left(\mathrm{SU}_{3}, \mathrm{SU}_{4}\right)$ content of the following representation of $\mathrm{SU}_{12}$,

$\square \rightarrow(\square,, \square)+(\square, \square \square)+(\cdot, \square)+(\square, \square)+(\square, \square)$

or

$$
(572) \rightarrow\left(10,20^{\prime}\right)+(8,20)+\left(1,20^{\prime}\right)+(8,4)+\left(8,20^{\prime}\right)
$$


Notice that the representation $(\square, \square, \square)$ of $\left(\mathrm{SU}_{3}, \mathrm{SU}_{4}\right)$ appears only once.

The tabulation of Table $C$ requires long calculations.* For higher orders we have used a computer.

We summarize the various -pplicatiors of the tables in the following diagram:

\begin{tabular}{|c|c|c|}
\hline TABIE & $\sum_{\mathrm{f}}^{\text {Symmetric Group }}$ & $\begin{array}{l}\text { Unitary Group } \\
\qquad \mathrm{SU}_{n}\end{array}$ \\
\hline A & Dimension & Dimension \\
\hline \multirow{2}{*}{ B } & \multirow{2}{*}{$\begin{array}{l}\text { Decomposition of outer product } \\
\left(\sum_{f_{1}}, \sum f_{2}\right) \rightarrow \sum f_{1}+f_{2}\end{array}$} & $\left(\mathrm{SU}_{\mathrm{m}}, \mathrm{SU}_{\mathrm{n}}\right)$ content of $\mathrm{SU}_{\mathrm{m}+\mathrm{n}}$ \\
\hline & & Clebsch-Gordan series for $\mathrm{SU}_{n}$ \\
\hline C & $\begin{array}{l}\text { Clebsch-Gordan series of } \\
\qquad f\end{array}$ & $\left(\mathrm{SU}_{\mathrm{m}}, \mathrm{SU}_{\mathrm{n}}\right)$ content of $\mathrm{SU}_{\mathrm{mn}}$ \\
\hline
\end{tabular}

* Some explicit formulas can be found, for instance, in Ref. 4. or can be computed using Frobenius' formula for the characters of the symmetric group. The most straightforward method uses the orthogonality of characters. 


\begin{tabular}{|c|c|c|c|c|c|c|c|c|c|c|c|}
\hline & $\sum$ & $\mathrm{SU}_{3}$ & SU. & $\mathrm{SU}_{5}$ & ${ }_{6}$ & का & $\mathrm{SU}_{B}$ & $\mathrm{SU}_{9}$ & $\mathrm{SU}_{20}$ & $\mathrm{SU}_{21}$ & $\mathrm{SU}_{12}$ \\
\hline (1) & 1 & 3 & 4 & 5 & 6 & 7 & 8 & 9 & 10 & II & 12 \\
\hline (2) & 1 & 6 & 10 & 15 & 21 & 28 & 36 & 45 & 55 & 66 & 78 \\
\hline$\left(1^{2}\right)$ & 1 & 3 & 6 & 10 & 15 & 21 & 28 & 36 & 45 & 55 & 66 \\
\hline (3) & 1 & 10 & 20 & 35 & 56 & 84 & 120 & 165 & 220 & 286 & 364 \\
\hline$(?, 1)$ & 2 & 9 & 20 & 40 & 70 & $i=2$ & 208 & $\therefore 0$ & 330 & 440 & 572 \\
\hline$\left(1^{3}\right)$ & 1 & 1 & $i$ & 20 & $=$ & $y$ & 56 & 34 & 120 & 165 & 220 \\
\hline (4) & 1 & 15 & 35 & 70 & 126 & 210 & 330 & 495 & 715 & 1001 & 1365 \\
\hline$(3,1)$ & 3 & 15 & 45 & 105 & 210 & 378 & 630 & 990 & 1485 & 2145 & 3003 \\
\hline$\left(2^{2}\right)$ & 2 & 6 & 20 & 50 & 105 & 196 & 336 & 540 & 825 & 1210 & 1716 \\
\hline$\left(2,1^{2}\right)$ & 3 & 3 & 15 & 45 & 105 & 210 & 378 & 630 & 990 & 1485 & 2145 \\
\hline$\left(1^{4}\right)$ & 1 & $*$ & 1 & 5 & 15 & 35 & 70 & 126 & 210 & 330 & 495 \\
\hline (5) & $I$ & 21. & 56 & 126 & 252 & $46 e$ & 792 & 1287 & 2002 & 3003 & 4368 \\
\hline$(4,1)$ & 4 & 24 & 84 & 224 & 504 & 1008 & 1348 & 3268 & 5148 & 8008 & 12012 \\
\hline$(3,2)$ & 5 & 15 & 60 & 175 & 420 & 882 & 1680 & $\Leftrightarrow 7 ?$ & 4950 & 7865 & 12012 \\
\hline$\left(3,1^{2}\right)$ & 6 & 6 & 36 & 126 & 336 & 756 & 1512 & 2772 & 4752 & $\pi 722$ & 12012 \\
\hline$\left(2^{2} 1\right)$ & 5 & 3 & 20 & 75 & 220 & 490 & 1008 & 1890 & 3300 & 5445 & 8580 \\
\hline$\left(2,1^{3}\right)$ & 4 & * & 4 & 24 & 84 & 224 & 504 & 1008 & 1848 & 3168 & 5148 \\
\hline$\left(1^{5}\right)$ & 1 & * & * & 1 & 6 & 21 & 56 & 126 & 252 & 462 & 792 \\
\hline (6) & 1 & 28 & 84 & 210 & 462 & 924 & 1716 & 3003 & 5005 & 8008 & 12376 \\
\hline$(5,1)$ & 5 & 35 & 140 & 420 & 1050 & 2310 & 4620 & 8580 & 15015 & 25025 & 40040 \\
\hline$(4,2)$ & 9 & 27 & 126 & 420 & 2134 & 2646 & 5544 & 10692 & 19305 & 33033 & 54054 \\
\hline$\left(4,1^{2}\right)$ & 10 & 10 & 70 & 280 & 840 & 2100 & 4620 & 9240 & 17160 & 30030 & 50050 \\
\hline$\left(3^{2}\right)$ & 5 & 10 & 50 & 175 & 490 & 1176 & 2520 & 4950 & 9075 & 15730 & 26026 \\
\hline$(3,2,1)$ & 16 & 8 & 64 & 280 & 896 & 2352 & 5376 & 11088 & 21120 & 37752 & 64064 \\
\hline$\left(3,1^{3}\right)$ & 10 & * & 10 & 70 & 280 & 840 & 2100 & 4620 & 9240 & 17160 & 30030 \\
\hline$\left(2^{3}\right)$ & 5 & 1 & 10 & 50 & 175 & 490 & 2276 & 2520 & 4950 & 9075 & 15730 \\
\hline$\left(2^{2} 1^{2}\right)$ & 9 & * & 6 & 45 & 189 & 588 & 1512 & 3402 & 6930 & 13068 & 23166 \\
\hline$\left(2,1^{4}\right)$ & 5 & * & * & 5 & 35 & 140 & 420 & 1050 & 2310 & 4620 & 8580 \\
\hline$\left(1^{6}\right)$ & 1 & * & * & * & 1 & 7 & 28 & 84 & 210 & 462 & 924 \\
\hline (7) & 1 & 36 & 120 & 330 & 792 & 1716 & 3432 & 6435 & 11440 & 19448 & 31824 \\
\hline$(6,1)$ & 6 & 48 & 216 & 720 & 1980 & 4752 & 10296 & 20592 & 38610 & 68640 & 116688 \\
\hline$(5,2)$ & 14 & 42 & 224 & 840 & 2520 & 6468 & 14784 & 30888 & 60060 & 110110 & 192192 \\
\hline$\left(5,1^{2}\right)$ & 15 & 15 & 120 & 540 & 1800 & 4950 & 11880 & 25740 & $51 \div 30$ & 96525 & 171600 \\
\hline$(4,3)$ & 14 & 24 & 140 & 560 & 1764 & 4704 & 21088 & 23760 & 47190 & 88088 & 156156 \\
\hline$(4,2,1)$ & 35 & 15 & 140 & 700 & 2520 & 7350 & 18480 & $41580^{\circ}$ & 85800 & 165165 & 300300 \\
\hline$\left(4,1^{3}\right\}$ & 20 & * & 20 & 160 & 720 & 2400 & 6600 & 15840 & 34320 & 68640 & 128700 \\
\hline$\left(3^{2}, 1\right)$ & 21 & 6 & 60 & 315 & 2176 & 3528 & 9072 & 20790 & 43560 & 84942 & 156156 \\
\hline$\left(3,2^{2}\right)$ & 21 & 3 & 36 & 210 & 840 & 2646 & 7056 & 16632 & 35640 & 70785 & 132132 \\
\hline$\left(3,2,1^{2}\right)$ & 35 & $\star$ & 20 & 175 & 840 & 2940 & 8400 & 20790 & 46200 & 94380 & 180180 \\
\hline$\left(3,1^{4}\right)$ & 15 & $*$ & $*$ & 15 & 120 & 540 & 1800 & 4950 & 11880 & 25740 & 52480 \\
\hline$\left(2^{3}, 1\right)$ & 14 & * & 4 & 40. & 210 & 784 & 2352 & 6048 & 13860 & 29040 & 56628 \\
\hline$\left(2^{2} 1^{3}\right)$ & 14 & * & $*$ & 30 & 84 & 392 & 2344 & 3780 & 9240 & 20328 & 41284 \\
\hline
\end{tabular}


TABLE-A (Continued)

\begin{tabular}{|c|c|c|c|c|c|c|c|c|c|c|c|}
\hline & $\sum$ & $\mathrm{SU}_{3}$ & $\mathrm{SU}_{4}$ & $\mathrm{SU}_{5}$ & $\mathrm{SU}_{5}$ & $\mathrm{Su}_{7}$ & $\mathrm{SU}_{8}$ & $\mathrm{SU}_{9}$ & $\mathrm{SU}_{10}$ & $\mathrm{su}_{11}$ & $\mathrm{su}_{12}$ \\
\hline$\left(2,1^{5}\right)$ & 6 & * & * & * & 6 & 48 & 216 & 720 & 1980 & 4752 & 10296 \\
\hline$\left(1^{7}\right)$ & 1 & * & * & * & * & 1 & 8 & 36 & 120 & 330 & 792 \\
\hline (8) & 1 & 45 & 165 & 495 & 1287 & 3003 & 6435 & 12870 & 24310 & 43758 & 75582 \\
\hline$(7,1)$ & 7 & 63 & 315 & 1155 & 3465 & 9009 & 21021 & 45045 & 90090 & 170170 & 306306 \\
\hline$(6,2)$ & 20 & 6o & 360 & 1500 & 4,50 & $1 ; 060$ & $31: 20$ & $772=0$ & 160875 & 314600 & 583440 \\
\hline$\left(6,1^{2}\right)$ & 21 & 21 & 109 & 945 & 3465 & 1039 , & $2: 327$ & 63063 & 135135 & 270270 & 510510 \\
\hline$(5,3)$ & 28 & 42 & 280 & 1260 & 4410 & 12936 & 33264 & 77220 & 165165 & 330330 & 624624 \\
\hline$(5,2,1)$ & 64 & 24 & 256 & 1440 & 5760 & 18480 & 50688 & 123552 & 274560 & 566280 & 1098240 \\
\hline$\left(5,1^{3}\right)$ & 35 & * & 35 & 315 & 1575 & 5775 & 17325 & 45045 & 105105 & 225225 & 450450 \\
\hline$\left(4^{2}\right)$ & 14 & 15 & 105 & 490 & 1764 & 5292 & 13860 & 32670 & 70785 & 143143 & 273273 \\
\hline$(4,3,1)$ & 70 & 15 & $i 75$ & 1050 & 4410 & 14700 & 41580 & 103950 & 235950 & 495495 & 975975 \\
\hline$\left(4,2^{2}\right)$ & 55 & 6 & 84 & 560 & 2520 & 8820 & 25872 & 65528 & 154440 & 330330 & 660660 \\
\hline$\left(4,2,1^{2}\right)$ & 90 & * & 45 & 450 & 2430 & 9450 & 29700 & RC190 & 193050 & 424710 & 868725 \\
\hline$\left(4,1^{4}\right)$ & 35 & * & * & 35 & 315 & 1575 & 5775 & 17325 & 45045 & 105105 & 225225 \\
\hline$\left(3^{2} 1^{2}\right)$ & 42 & 3 & 45 & 315 & 1470 & 5292 & 15876 & 41580 & 98010 & 212355 & 429429 \\
\hline$\left(3^{2} 1^{2}\right)$ & 56 & * & 20 & 210 & 1176 & 4704 & 15120 & 41580 & 101640 & 226512 & 468468 \\
\hline$\left(3,2^{2} 1\right)$ & 70 & * & 15 & 175 & 1050 & 4410 & 14700 & 41580 & 103950 & 235950 & 495490 \\
\hline$\left(3,2,1^{3}\right)$ & 64 & * & * & 40 & 384 & 2016 & 7680 & 23760 & 63360 & 151008 & 329472 \\
\hline$\left(3,1^{5}\right)$ & 21 & * & * & * & 21 & 189 & 945 & 3465 & 10395 & 27027 & 63063 \\
\hline$\left(2^{4}\right)$ & 14 & * & 1 & 15 & 105 & 490 & 1764 & 5292 & 13860 & 32670 & 70785 \\
\hline$\left(2^{3} 1^{2}\right)$ & 28 & * & * & 10 & 105 & 588 & 2352 & 7560 & 20790 & 50820 & 113256 \\
\hline$\left(2^{2} 1^{4}\right)$ & 20 & * & $*$ & * & 15 & 140 & 720 & 2700 & 8250 & 21780 & 51480 \\
\hline$\left(2,1^{6}\right)$ & 7 & * & * & * & * & 7 & 63 & 315 & 1155 & 3465 & 9009 \\
\hline$\left(1^{8}\right)$ & 1 & * & * & * & * & * & 1 & 9 & 45 & 165 & 495 \\
\hline
\end{tabular}




$$
\begin{array}{|l|c|c|}
\hline f+f_{2}=2 & i 2 i & (1 z) \\
\hline(1)(1) & 1 & 1 \\
\hline
\end{array}
$$

\begin{tabular}{|c|c|c|c|}
\hline$f_{1}+f_{2}=3$ & $(3)$ & $(2,1)$ & $\left(1^{3}\right)$ \\
\hline$(1)(2)$ & 1 & 1 & \\
\hline$(1)\left(1^{2}\right)$ & & 1 & 1 \\
\hline
\end{tabular}

\begin{tabular}{|l|l|c|c|c|c|c|}
\hline & $f_{1}+f_{2}=4$ & $(4)$ & $(3,1)$ & $\left(2^{2}\right)$ & $\left(2,1^{2}\right)$ & $\left(1^{4}\right)$ \\
\hline $\begin{array}{c}m \\
+\end{array}$ & $(1)(3)$ & 1 & 1 & & & \\
\hline $\begin{array}{c}c \\
+ \\
N\end{array}$ & $\frac{(1)(2,1)}{(1)\left(1^{3}\right)}$ & & 1 & 1 & 1 & \\
\hline & $\frac{(2)\left(1^{2}\right)}{\left(1^{2}\right)\left(1^{2}\right)}$ & 1 & 1 & 1 & & 1 \\
\hline
\end{tabular}


TABLE B

(Continued)

\begin{tabular}{|c|c|c|c|c|c|c|c|c|}
\hline & $f_{1}+f_{2}=:$ & (5) & $(4,2)$ & $(3, \ldots)$ & $\left(3,2^{2}\right)$ & $(2,1)$ & $\left(2,1^{3}\right)$ & $\left(1^{5}\right)$ \\
\hline \multirow{5}{*}{$\begin{array}{c}+ \\
+ \\
+\end{array}$} & (I) (4) & 1 & 1 & & & & & \\
\hline & (1) $(3,1)$ & & 1 & 1 & 1 & & & \\
\hline & (1) $\left(2^{2}\right)$ & & & 1 & & 1 & & \\
\hline & (1) $\left(2,1^{2}\right)$ & & & & 1. & 1 & 1 & \\
\hline & $(1)\left(I^{4}\right)$ & & & & & & 1 & 1 \\
\hline \multirow{6}{*}{$\begin{array}{l}m \\
+ \\
\text { cs }\end{array}$} & (2) $(3)$ & $I$ & 1 & 1 & & & & \\
\hline & (2) $(2,1)$ & & 1 & 1 & 1 & 1 & & \\
\hline & (2) $\left(1^{3}\right)$ & & & & 1 & & 1 & \\
\hline & $\left(1^{2}\right)(3)$ & & 1 & & 1 & & & \\
\hline & $\left(1^{2}\right)(2,1)$ & & & 1 & $i$ & 1 & 1 & \\
\hline & $\left(1^{2}\right)\left(1^{3}\right)$ & & & & & 1 & 1 & 1 \\
\hline
\end{tabular}


TABIE B (continued)

\begin{tabular}{|c|c|c|c|c|c|c|c|c|c|c|c|c|}
\hline \multirow{8}{*}{$\begin{array}{l}\text { nn } \\
+ \\
-1\end{array}$} & $f_{1}+f_{2}=6$ & $(6)$ & $(5.1)$ & $(4,2)$ & $\left(4,1^{2}\right)$ & $\left(3^{2}\right)$ & $(3,2,1)$ & $\left(2^{3}\right)$ & $\left(3,1^{3}\right)$ & $\left(2^{2}, 1^{2}\right)$ & $\left(2,1^{4}\right)$ & $\left(1^{6}\right)$ \\
\hline & $(1)(5)$ & $I$ & 2 & & & & & & & & & \\
\hline & (1) $(4,1)$ & & 1 & 1 & 1 & & & & & & & \\
\hline & $(1)(3,2)$ & & & 1 & & 1 & 1 & & & & & \\
\hline & $(1)\left(3,1^{2}\right)$ & & & & 1 & & 1 & & 1 & & & \\
\hline & (1) $\left(2^{2}, 1\right)$ & & & & & & 1 & 1 & & 1 & & \\
\hline & (1) $\left(2,1^{3}\right)$ & & & & & & & & 1 & 1 & 1 & \\
\hline & $(1)\left(1^{5}\right)$ & & & & & & & & & & 1 & 1 \\
\hline \multirow{10}{*}{$\begin{array}{l}+ \\
+ \\
\sim\end{array}$} & $(2)(4)$ & 1 & 1 & 1 & & & & & & & & \\
\hline & $(2)(3,1)$ & & 1 & 1 & $I$ & 1 & 1 & & & & & \\
\hline & $(2)\left(2^{2}\right)$ & & & 1 & & & I & 1 & & & & \\
\hline & $(2)\left(2,1^{2}\right)$ & & & & 1 & & $I$ & & $I$ & 1 & & \\
\hline & $(2)\left(1^{4}\right)$ & & & & & & & & 1 & & 1 & \\
\hline & $\left(1^{2}\right)(4)$ & & 1 & & 1 & & & & & & & \\
\hline & $\left(1^{2}\right)(3,1)$ & & & 1 & 1 & & 1 & & 1 & & & 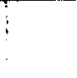 \\
\hline & $\left(1^{2}\right)\left(2^{2}\right)$ & & & & & 1 & 1 & & & 1 & & 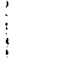 \\
\hline & $\left(1^{2}\right)\left(2,1^{2}\right)$ & & & & & & $i$ & 1 & 1 & 1 & 1 & $\vdots$ \\
\hline & $\left(1^{2}\right)\left(1^{4}\right)$ & & & & & & & & & 1 & 1 & 1 \\
\hline
\end{tabular}


TABLE B (continued)

\begin{tabular}{|l|c|c|c|c|c|c|c|c|c|c|c|c|}
\hline$f_{1}+f_{2}=6$ & $(6)$ & $(5,1)$ & $(4,2)$ & $\left(4,1^{2}\right)$ & $\left(3^{2}\right)$ & $(3,2,1)$ & $\left(2^{3}\right)$ & $\left(3,1^{3}\right)$ & $\left(2^{2}, 1^{2}\right)$ & $\left(2,1^{4}\right)$ & $\left(1^{5}\right)$ \\
\hline$m$ \\
\hline$m$ \\
\hline$m$
\end{tabular}




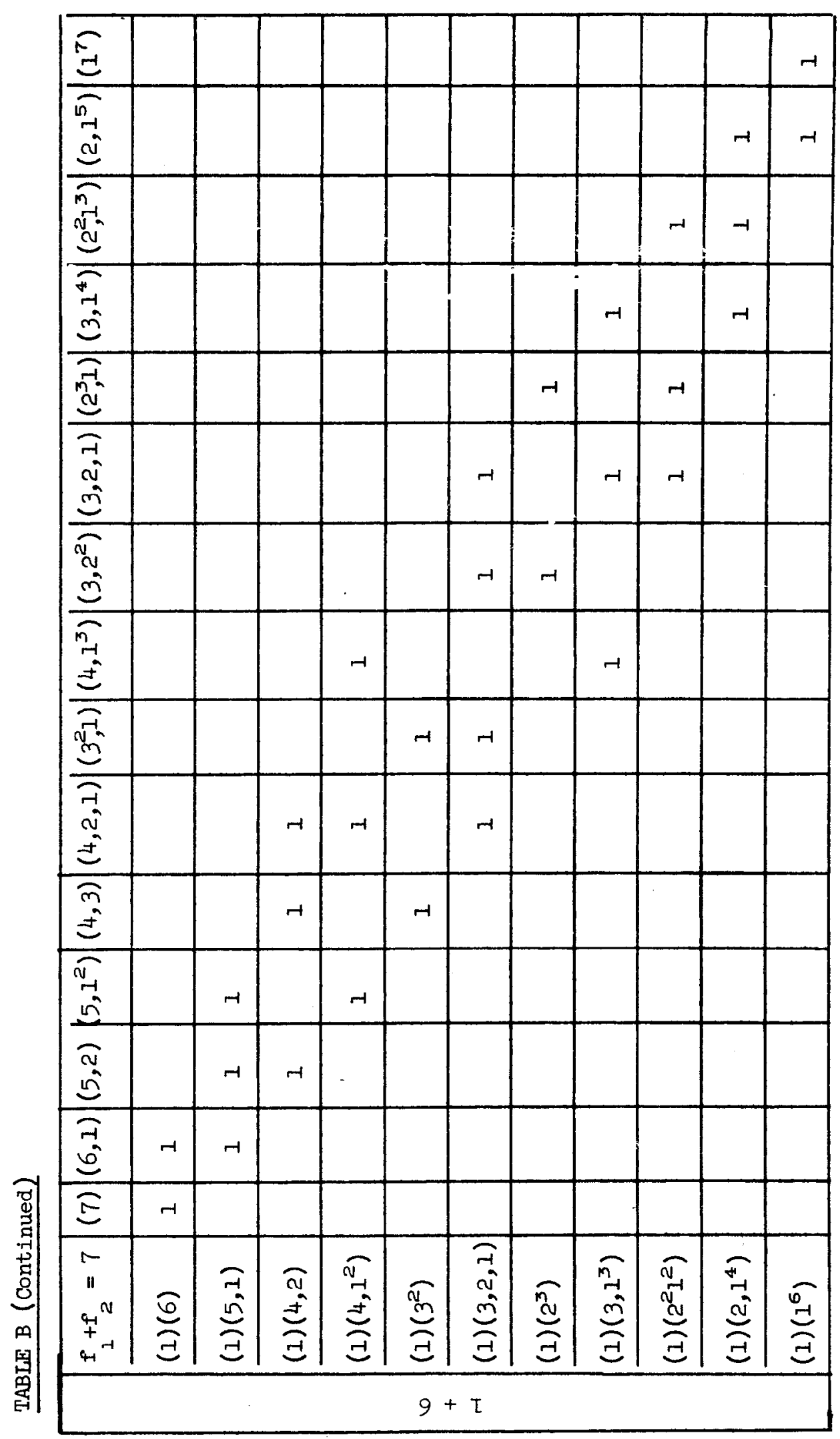




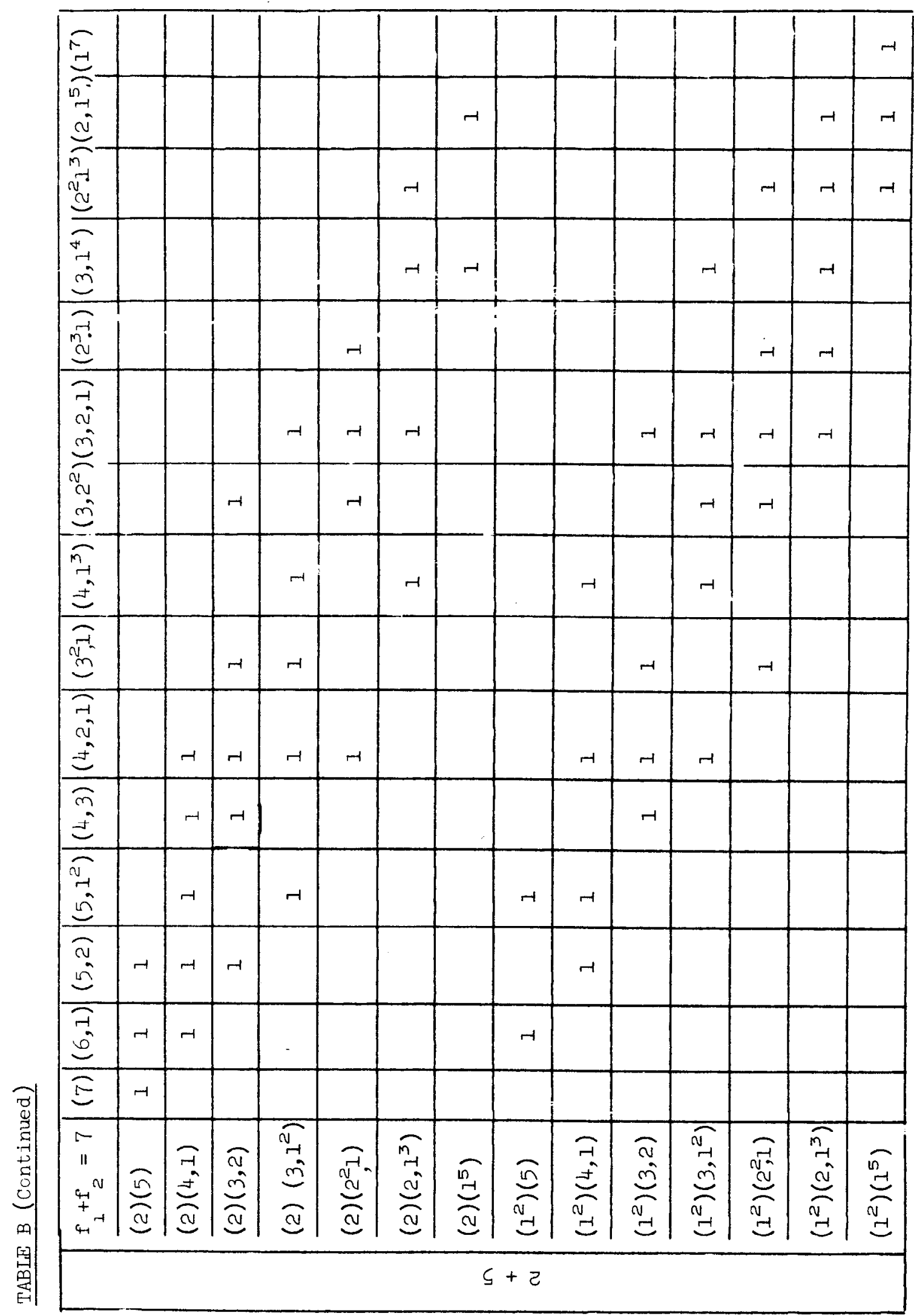




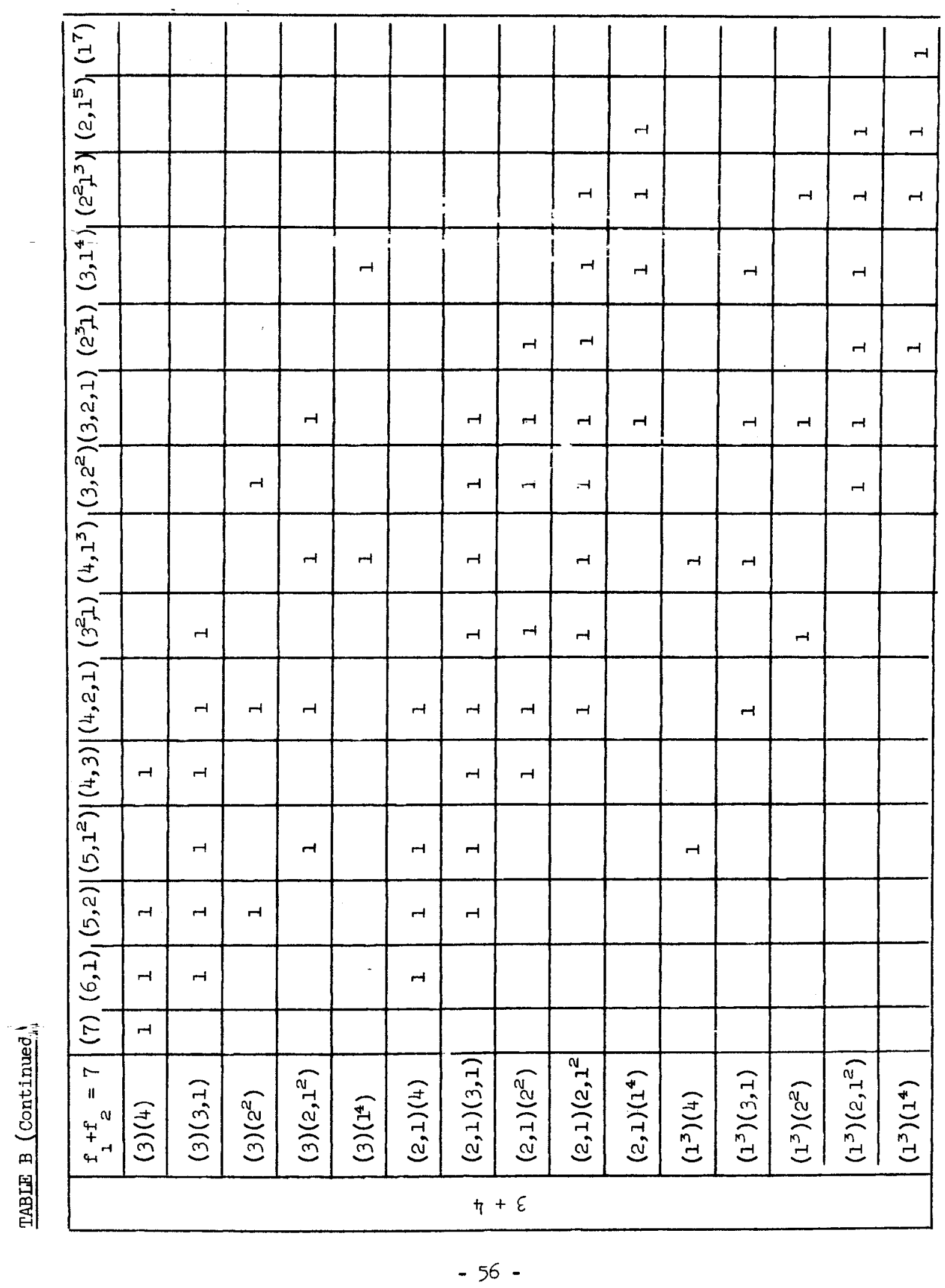


TABIE B (Continued)

\begin{tabular}{|c|c|c|c|c|c|c|c|c|c|c|c|c|c|c|c|c|c|c|c|c|c|c|c|}
\hline \multirow{16}{*}{$\begin{array}{r}5 \\
+ \\
-1\end{array}$} & $f_{1}+f_{2}=8$ & $\widehat{\infty}$ & 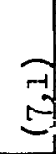 & & $\begin{array}{l}\widetilde{v} \\
t \\
v^{-}\end{array}$ & m & 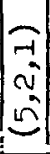 & $\mid \begin{array}{c}m \\
-10\end{array}$ & $\widetilde{T}$ & 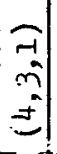 & \begin{tabular}{l}
$\widetilde{N}$ \\
\multirow{N}{*}{} \\
\pm \\
\pm
\end{tabular} & 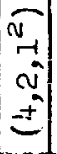 & 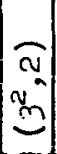 & $\begin{array}{c}- \\
w \\
- \\
w \\
m\end{array}$ & & $\stackrel{-}{\mathbb{N}}$ & $\begin{array}{l}- \\
\pi \\
= \\
\pm \\
\pm\end{array}$ & 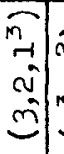 & & & $\begin{array}{c}\pi \\
-1 \\
c \\
c \\
c\end{array}$ & & $\mathscr{I}$ \\
\hline & $(1)(7)$ & i & 1 & & & & & & & & & & & & & & & & & & & & \\
\hline & $(1)(6,1)$ & & 1 & 1 & 1 & & & & & & & & & & & & & & & & & & \\
\hline & $(1)(5,2)$ & & & 1 & & 1 & 1 & & & & & & & & & & & & & & & & \\
\hline & (1) $\left(5,1^{2}\right)$ & & & & 1 & & 1 & 1 & & & & & & & & & & & & & & & \\
\hline & $(1)(4,3)$ & & & & & 1 & & & 1 & 1 & & & & & & & & & & & & & \\
\hline & $(1)(4,2,1)$ & & & & & & 1 & & & 1 & 1 & 1 & & & & & & & & & & & \\
\hline & $(1)\left(3^{2}, 1\right)$ & & & & & & & & & 1 & & & 1 & 1 & & & & & & & & & \\
\hline & $(1)\left(4,1^{3}\right)$ & & & & & & & 1 & & & & 1 & & & & & 1 & & & & & & \\
\hline & (I) $\left(3,2^{2}\right)$ & & & & & & & & & & 1 & & 1 & & 1 & & & & & & & & \\
\hline & (1) $\left(3,2,1^{2}\right)$ & & & & & & & & & & & 1 & & 1 & 1 & & & 1 & & & & & \\
\hline & $(1)\left(2^{3} 1\right)$ & & & & & & & & & & & & & & 1 & 1 & & & $I$ & & & & \\
\hline & $(1)\left(3,1^{4}\right)$ & & & & & & & & & & & & & & & & 1 & 1 & & 1 & & & \\
\hline & $(1)\left(2^{2}, 1^{3}\right)$ & & & & & & & & & & & & & & & & & 1 & 1 & & 1 & & \\
\hline & $(1)\left(2,1^{5}\right)$ & & & & & & & & & & & & & & & & & & & 1 & 1 & 1 & \\
\hline & $(1)\left(1^{7}\right)$ & & & & & & & & & & & & & & & & & & & & 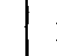 & 1 & 1 \\
\hline & $(2)(6)$ & 1 & 1 & 1 & & & & & & & & & & & & & & & & & & & \\
\hline 6 & $(2)(5,1)$ & & $I$ & 1 & 1 & 1 & 1 & & & & & & & & & & & & & & & & \\
\hline cu & $(2)(4,2)$ & & & 1 & & 1 & 1 & & 1 & 1 & 1 & & & & & & & & & & & & \\
\hline & (2) $\left(4,1^{2}\right)$ & & & & 1 & & 1 & 1 & & 1 & & 1 & & & & & & & & & & & \\
\hline
\end{tabular}


TABLE B (Continued)

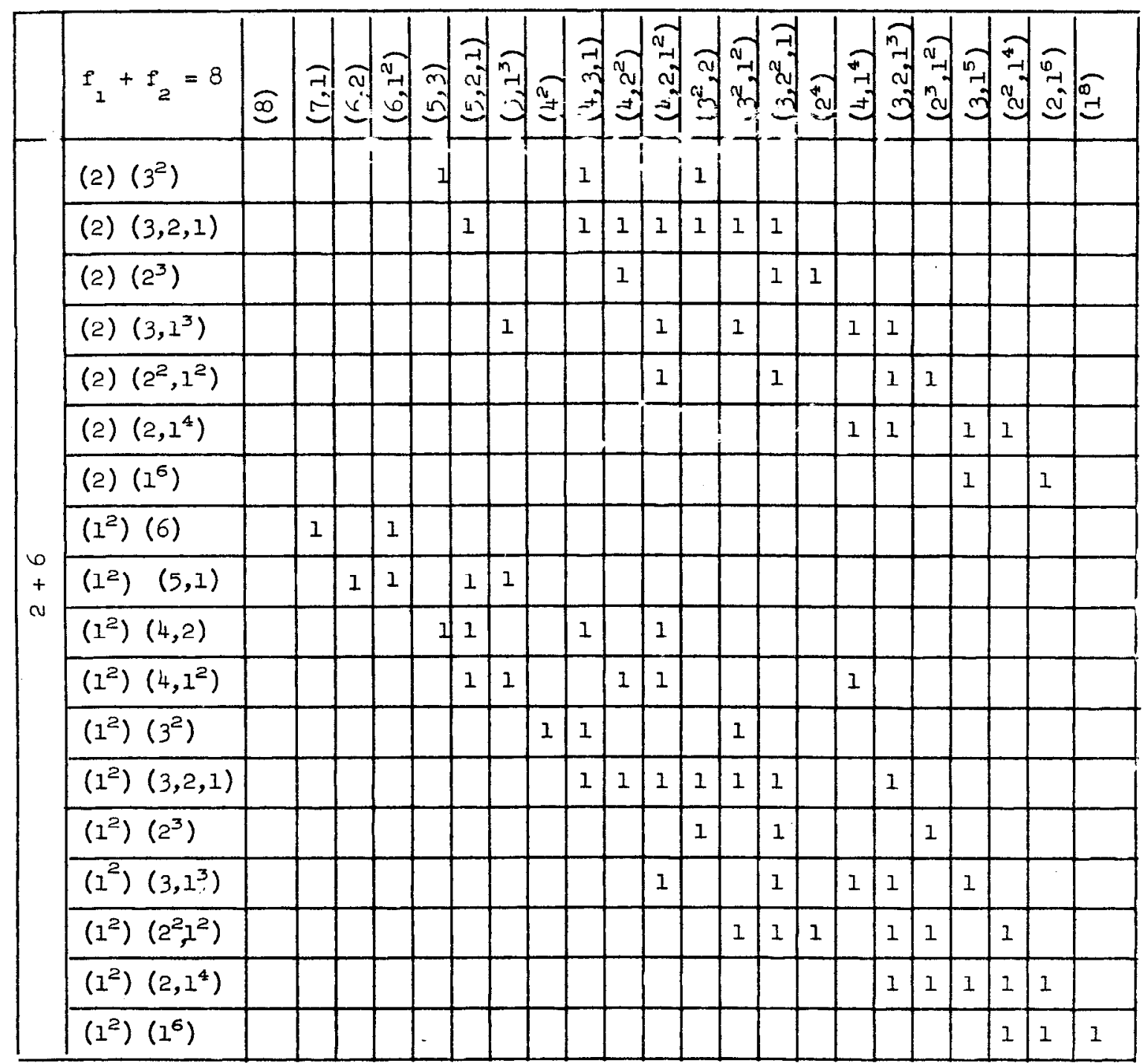


TABIE B (Continued)

\begin{tabular}{|c|c|c|c|c|c|c|c|c|c|c|c|c|c|c|c|c|c|c|c|c|c|c|}
\hline$f_{1}+f_{2}=8$ & $\infty$ & 5 & 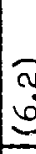 & $\begin{array}{l}\pi \\
-1 \\
0 \\
0\end{array}$ & $\stackrel{m}{\omega}$ & $\mid \begin{array}{c}-1 \\
0 \\
0 \\
5\end{array}$ & $\begin{array}{l}-1 \\
-1 \\
0 \\
0\end{array}$ & & $\begin{array}{l}\vec{f} \\
m \\
\pm \\
\pm\end{array}$ & 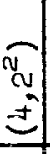 & 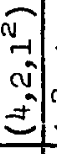 & 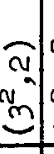 & कू & 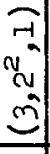 & $\underset{v}{\stackrel{N}{N}}$ & $\begin{array}{l}- \\
- \\
\pm \\
\pm\end{array}$ & 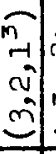 & 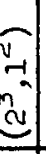 & $\begin{array}{c}-1 \\
n \\
-1 \\
-1\end{array}$ & 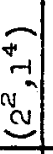 & $\begin{array}{c}0 \\
\stackrel{0}{a} \\
\stackrel{-}{0}\end{array}$ & \\
\hline (3) (5) & 1 & I & 1 & & 1 & & & & . & & & & & & & & & & & & & \\
\hline$(3 ;(4,1)$ & & 1 & 1 & 1 & 1 & 1 & & 1 & $\because$ & & & & & & & & & & & & & \\
\hline (3) $(3,2)$ & & & 1 & & 1 & 1 & & & 1 & 1 & . & 1 & & & & & & & & & & \\
\hline (3) $\left(3,1^{2}\right)$ & & & & 1 & & 1 & 1 & & I & & 1 & & 1 & & & & & & & & & \\
\hline (3) $\left(2^{2}, 1\right)$ & & & & & & 1 & & & & 1 & 1 & & & 1 & & & & & & & & \\
\hline (3) $\left(2,1^{3}\right)$ & & & & & & & 1 & & & & 1 & & & & & 1 & 1 & & & & & \\
\hline (3) $\left(1^{5}\right)$ & & & & & & & & & & & & & & & & 1 & & & 1 & & & \\
\hline$(2,1)(5)$ & & 1 & 1 & 1 & & 1 & & & & & & & & & & & & & & & & \\
\hline$(2,1)(4,1)$ & & & 1 & 1 & 1 & 2 & 1 & & 1 & 1 & 1 & & & & & . & & & & & & \\
\hline$(2,1)(3,2)$ & & & & & 1 & 1 & & 1 & 2 & 1 & 1 & 1 & 1 & 1 & & & & & & & & \\
\hline$(2,1)\left(3,1^{2}\right)$ & & & & & & 1 & 1 & & 1 & 1 & 2 & 1 & 1 & 1 & & 1 & 1 & & & & & \\
\hline$(2,1)\left(2^{2}, 1\right)$ & & & & & & & & & 1 & 1 & 1 & 1 & 1 & 2 & 1 & & $1:$ & 1 & & & & \\
\hline$(2,1)\left(2,1^{3}\right)$ & & & & & & & & & & & 1 & & 1 & 1 & & 1 & 2 & 1 & 1 & 1 & & \\
\hline$(2,1)\left(1^{5}\right)$ & & & & & & & & & & & & & & & & & 1 & & 1 & 1. & 1 & \\
\hline$\left(1^{3}\right)(5)$ & & & & 1 & & & 1 & & & & & & & & & & & & & & & \\
\hline$\left(1^{3}\right) \quad(4,1)$ & & & & & & 1 & 1 & & & & 1 & & & & & 1 & & & & & & \\
\hline$\left(1^{3}\right)(3,2)$ & & & & & & & & & 1 & & 1 & & 1 & & & & 1 & & & & & \\
\hline$\left(1^{3}\right)\left(3,1^{2}\right)$ & & & & & & & & & & 1 & 1 & & & 1 & & 1 & 1 & & 1 & & & \\
\hline$\left(1^{3}\right)\left(2^{2}, 1\right)$ & & & & & & & & & & & & 1 & 1 & 1 & & & 1 & 1 & & 1 & & \\
\hline$\left(1^{3}\right)\left(2,1^{3}\right)$ & & & & & & & & & & & & & & 1 & 1 & & 1 & 1 & 1 & 1 & 1 & \\
\hline$\left(1^{3}\right)\left(1^{5}\right)$ & & & & & & & & & & & & & & & & & & $1 \mid$ & & 1 & 1 & 1 \\
\hline
\end{tabular}


TABLE B (Continued)

\begin{tabular}{|c|c|c|c|c|c|c|c|c|c|c|c|c|c|c|c|c|c|c|c|c|}
\hline & $f_{1}+f_{2}=8$ & $\widehat{\infty}$ & $\begin{array}{c}-1 \\
- \\
-\end{array}$ & 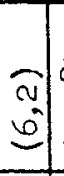 & $\begin{array}{l}-1 \\
x_{-1} \\
6 \\
0\end{array}$ & ñ & 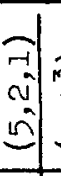 & & $\underset{d}{0}$ & 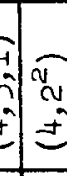 & {$\left[\begin{array}{c}\tilde{N} \\
-1 \\
0 \\
\vdots \\
\dot{U}\end{array}\right]$} & 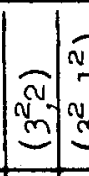 & 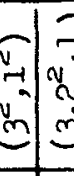 & 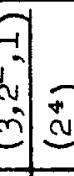 & $\mid \begin{array}{l}5 \\
5-1 \\
0 \\
\pm\end{array}$ & $\begin{array}{c}n \\
-1 \\
0 \\
0 \\
m\end{array}$ & & & i & $\stackrel{0}{=}$ \\
\hline \multirow{15}{*}{$\begin{array}{l}+ \\
+ \\
+\end{array}$} & (4) (4) & 1 & 1 & 1 & & 1 & & & $I$ & & & & & & & & & & & \\
\hline & $(4)(3,1)$ & & 1 & 1 & 1 & 1 & 1. & & 1 & & & & & & & & & & & \\
\hline & $(4)\left(2^{2}\right)$ & & & 1 & & & 1 & & & 1 & & & & & & & & & & \\
\hline & $(4)\left(2,1^{2}\right)$ & & & & 1 & & 1 & 1 & & & 1 & & & & & & & & & \\
\hline & $(4)\left(1^{4}\right)$ & & & & & & & 1 & & & & & & & 1 & & & & & \\
\hline & $(3,1)(3,1)$ & & & 1 & 1 & 1 & 2 & 1 & 12 & 1 & 1 & 1 & 1 & & & & & & & \\
\hline & $(3,1)\left(2^{2}\right)$ & & & & & 1 & 1 & & 1 & 2 & 1 & 1 & 1 & & & & & & & \\
\hline & $(3,1)\left(2,1^{2}\right)$ & & & & & & 1 & 1 & 1 & + & 2 & & 11 & & 1 & 1 & & & & \\
\hline & $(3,1)\left(1^{4}\right)$ & & & & & & & & & & 1 & & & & 1 & 1 & 1 & & & \\
\hline & $\left(2^{2}\right)\left(2^{2}\right)$ & & & & & & & 1 & 1 & 1 & & & 11 & 11 & & & & & & \\
\hline & $\left(2^{2}\right)\left(2,1^{2}\right)$ & & & & & & & & 1 & & 1 & 1 & 1 & & & 1 & 1 & & & \\
\hline & $\left(2^{2}\right)\left(1^{4}\right)$ & & & & & & & & & & & & 1 & & & 1 & & 1 & & \\
\hline & $\left(2,1^{2}\right)\left(2,1^{2}\right)$ & & & & & & & & & 1 & 1 & 1 & 1 & 1 & 1 & 2 & 1 & 1 & & \\
\hline & $\left(2,1^{2}\right)\left(1^{4}\right)$ & & & & & & & & & & & & 1 & & & 1 & 1 & 1 & 1 & \\
\hline & $\left(1^{4}\right)\left(1^{4}\right)$ & & & & & & & & & & & & & 1 & & & 1 & & I & 1 \\
\hline
\end{tabular}




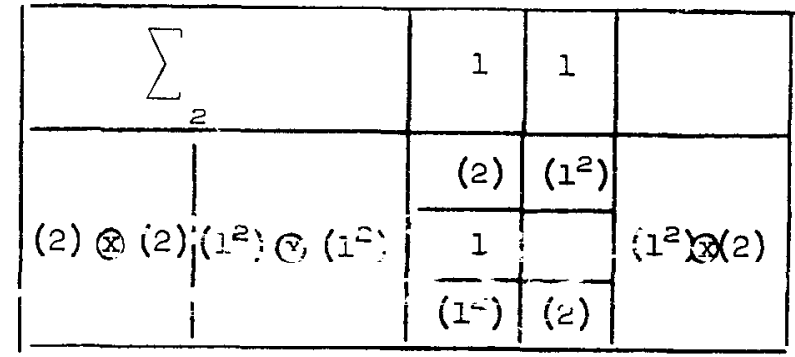

\begin{tabular}{|c|c|c|c|c|c|}
\hline$\sum_{3}$ & $\begin{array}{c}1 \\
(3)\end{array}$ & $\begin{array}{c}2 \\
(2,1)\end{array}$ & $\left(1^{3}\right)$ & \\
\hline$(3) \otimes(3)$ & $\left(1^{3}\right) \otimes\left(1^{3}\right)$ & 1 & & & $(3) \otimes\left(1^{3}\right)$ \\
\hline$(3) \otimes(2,1)$ & $\left(1^{3}\right) \otimes(2,1)$ & & 1 & & \\
\hline$(2,1) \otimes(2,1)$ & 1 & 1 & 1 & \\
\hline
\end{tabular}

\begin{tabular}{|l|l|c|c|c|c|c|}
\hline & 1 & 3 & 2 & 3 & 1 & \\
$(4)$ & $(3,1)$ & $\left(2^{2}\right)$ & $\left(2,1^{2}\right)$ & $\left(1^{4}\right)$ & \\
\hline$(4) \times(4)\left(1^{4}\right) \times\left(1^{4}\right)$ & 1 & & & & & $(4) x\left(1^{4}\right)$ \\
\hline$(4) \times(3,1)\left(1^{4}\right) \times\left(2,1^{2}\right)$ & & 1 & & & & $\left(1^{4}\right)(3)(3,1)(4) \times\left(2,1^{2}\right)$ \\
\hline$(4) \times\left(2^{2}\right)\left(1^{4}\right) \times\left(2^{2}\right)$ & & & 1 & & & \\
\hline$(3,1) \times\left(2^{2}\right)\left(2,1^{2}\right) \times\left(2^{2}\right)$ & & 1 & & 1 & & \\
\hline$\left(2^{2}\right) \times\left(2^{2}\right)$ & 1 & & 1 & & 1 & \\
\hline
\end{tabular}




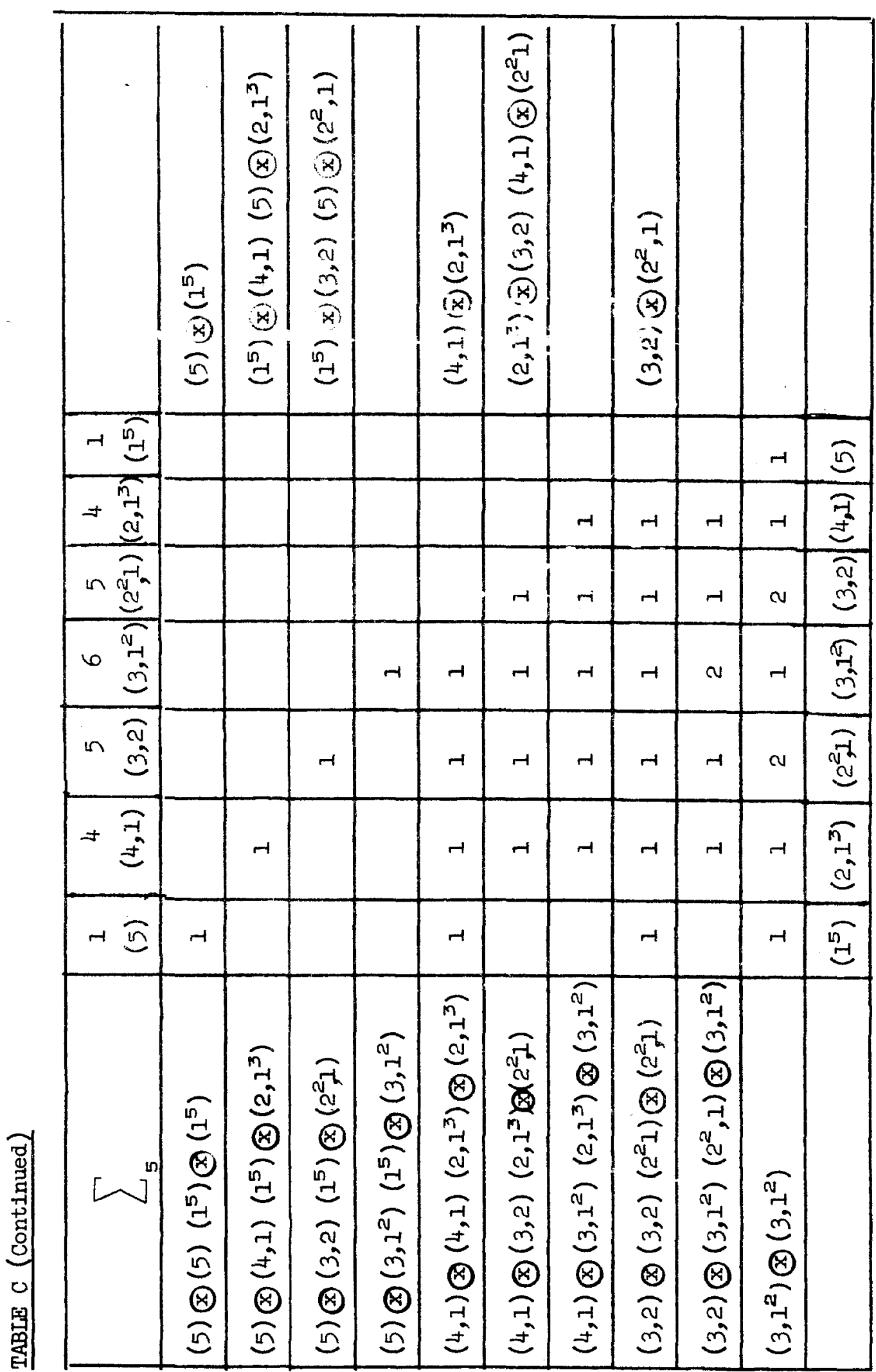


TABIE C (Continued)

\begin{tabular}{|c|c|c|c|c|c|c|c|c|c|c|c|c|c|}
\hline & $\sum_{6}$ & $\begin{array}{c}1 \\
(6)\end{array}$ & $\begin{array}{c}5 \\
(5,1)\end{array}$ & $\begin{array}{c}9 \\
(4,2)\end{array}$ & $\begin{array}{c}10 \\
\left(4,1^{2}\right)\end{array}$ & $\begin{array}{c}5 \\
\left(3^{2}\right)\end{array}$ & $\begin{array}{c}16 \\
(3,2,1)\end{array}$ & $\begin{array}{c}5 \\
\left(2^{3}\right)\end{array}$ & $\begin{array}{c}10 \\
\left(3,1^{3}\right)\end{array}$ & $\mid \begin{array}{c}9 \\
\left(2^{2} 1^{2}\right)\end{array}$ & $\mid \begin{array}{c}5 \\
\left(2,1^{4}\right)\end{array}$ & $\begin{array}{c}1 \\
\left(1^{6}\right)\end{array}$ & \\
\hline$(6) \otimes(6)$ & $\left(1^{6}\right) \otimes\left(1^{6}\right)$ & 1 & & & & & & & & & & & $(6) \otimes\left(1^{6}\right)$ \\
\hline$(6) \otimes(5,1)$ & $\left(1^{6}\right) \otimes\left(2,1^{4}\right)$ & & 1 & & & & & & & & & & $\left(1^{6}\right) \otimes(5,1)(6) \otimes\left(2,1^{4}\right)$ \\
\hline$(6) \otimes(4,2)$ & $\left(1^{6}\right) \otimes\left(2^{2}, 1^{2}\right)$ & & & 1 & & & & & & & & & $\left(1^{5}\right) \otimes(4,2)(6) \otimes\left(2^{2} 1^{2}\right)$ \\
\hline$(6) \otimes\left(4,1^{2}\right)$ & $\left(1^{6}\right) \otimes\left(3,1^{3}\right)$ & & & & 1 & & & & & & & & $\left(1^{6}\right) \otimes\left(4,1^{2}\right)(6) \otimes\left(3,1^{3}\right)$ \\
\hline$(6) \otimes\left(3^{2}\right)$ & $\left(1^{6}\right) \otimes\left(2^{3}\right)$ & & & & & 1 & & & & & & & $\left(1^{6}\right) \otimes\left(3^{2}\right)(6) \otimes\left(2^{3}\right)$ \\
\hline$(6) \otimes(3,2,1)$ & $\left(1^{6}\right) \otimes(3,2,1)$ & & & & & & 1 & & & & & & \\
\hline$(5,1) \otimes(5,1)$ & $\left(2,1^{4}\right) \otimes\left(2,1^{4}\right)$ & 1 & 1 & 1 & 1 & & & & & & & & $(5,1) \Omega\left(2,1^{4}\right)$ \\
\hline$(5,1) \otimes(4,2)$ & $\left(2,1^{4}\right) \otimes\left(2^{2} 1^{2}\right)$ & & 1 & 1 & 1 & 1 & 1 & & & & & & $\left(2,1^{4}\right) \otimes(6,2)(5,1) \otimes\left(2^{2}, 1^{2}\right)$ \\
\hline$(5,1) \otimes\left(4,1^{2}\right)$ & $\left(2,1^{4}\right) \otimes\left(3,1^{3}\right)$ & & 1 & 1 & 1 & & 1 & & 1 & & & & $\left(2,3^{4}\right) \otimes\left(4,1^{2}\right)(5,1) \otimes\left(3,1^{3}\right.$ \\
\hline$(5,1) \otimes\left(3^{2}\right)$ & $\left(2,1^{4}\right) \otimes\left(2^{3}\right)$ & & & 1 & & & 1 & & & & & & $\left(2,1^{4}\right) \otimes\left(3^{2}\right)(5,1) \otimes\left(2^{3}\right)$ \\
\hline$(5,1) \otimes(3,2,1)$ & $\left(2,1^{4}\right) \otimes(3,2,1)$ & & & 1 & 1 & 1 & 2 & 1 & 1. & 1 & & & \\
\hline$(4,2) @(1,2)$ & $\left(2^{2} 1^{2}\right) \otimes\left(2^{2}, 1^{2}\right)$ & 1 & 1 & 2 & 1 & & 2 & 1 & 1. & & & & $\left(A^{2}\right), \theta(4,2)$ \\
\hline$(4,2) \otimes\left(4,1^{2}\right)$ & $\left(2^{2}, 1^{2}\right) \otimes\left(3,1^{3}\right)$ & & 1 & 1 & 2 & 1 & 2 & & 1 & 1 & & & $\left(2^{2,2}\right) \otimes\left(4,1^{2}\right)(4,2) \otimes\left(3,1^{3}\right)$ \\
\hline$(4,2) \otimes\left(3^{2}\right)$ & $\left(2^{2}, 1^{2}\right) \otimes\left(2^{3}\right)$ & & 1 & & 1 & 1 & 1 & & & 1 & & & $\left(2^{2}, 2\right) \otimes\left(3^{2}\right)(4,2) \otimes\left(2^{3}\right)$ \\
\hline$(4,2) \otimes(3,2,1)$ & $\left(2^{2}, 1^{2}\right) \otimes(3,2,1)$ & & 1 & 2 & 2 & 1 & 3 & 1 & 2 & 2 & 1 & & \\
\hline$\left(4,1^{2}\right) \otimes\left(4,1^{2}\right)$ & $\left(3,1^{3}\right) \otimes\left(3,1^{3}\right)$ & 1 & 1 & 2 & 1 & 1 & 2 & 1 & 1 & 1 & 1 & & $\left(3,1^{3} ; 3\left(4,1^{2}\right)\right.$ \\
\hline$\left(4,1^{2}\right) \otimes\left(3^{2}\right)$ & $\left(3,1^{3}\right) \otimes\left(2^{3}\right)$ & & & 1 & 1 & & 1 & 1 & 1 & & & & $\left(1,1^{3}\right) \otimes\left(3^{2}\right)\left(4,1^{2}\right) \otimes\left(2^{3}\right)$ \\
\hline$\left(4,1^{2}\right) \Subset(3,2,1)$ & $\left(3,1^{3}\right) \circledast(3,2,1)$ & & 1 & 2 & 2 & 1 & 4 & 1 & 2 & 2 & 1 & & \\
\hline$\left(3^{2}\right) \times\left(3^{2}\right)$ & $\left(2^{3}\right)(x)\left(2^{3}\right)$ & 1 & & 1 & & & & 1 & $I$ & & & & $\left(2^{3}\right) @\left(3^{2}\right)$ \\
\hline$\left(3^{2}\right) \propto(3,2,1)$ & $\left(2^{3}\right) \otimes(3,2,1)$ & & 1 & 1 & 1 & $\therefore$ & 2 & & 3 & 1 & 1 & & \\
\hline \multicolumn{2}{|l|}{$(3,2,1) \otimes(3,2,1)$} & 1 & 2 & 3 & 4 & 2 & 5 & 2 & 4 & 3 & 2 & .1 & \\
\hline & & $\left(2^{6}\right)$ & $\left(2,1^{4}\right)$ & $\left(2^{2}, 1^{2}\right)$ & $\left(3,1^{3}\right)$ & $\left(2^{3}\right)$ & $(3,2,1)$ & $\left(3^{2}\right)$ & $\left(4,2^{2}\right)$ & $(4,2)$ & $(5,1)$ & (6) & \\
\hline
\end{tabular}


TABLE C (Continued)

\begin{tabular}{|c|c|c|c|c|c|c|c|c|c|c|c|c|c|c|c|}
\hline$\sum_{7}$ & 1 & 6. & 14 & 25 & 143 & $35 \mid 2$. & & & & 35 & 14 & 514 & 6 & 1 & \\
\hline & $E$ & 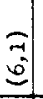 & & & है & & & & & ה: & & $\begin{array}{c}n \\
n=1 \\
0 \\
0\end{array}$ & 要 & $\approx$ & \\
\hline$\left(1^{7}\right)\left(1^{7}\right)$ & 2 & & & & & & & & & & & & & & $(7)\left(1^{7}\right)$ \\
\hline$\left(1^{7}\right)(2,15)$ & & 1 & & & & & & & & & & & & & $(7)\left(2,2,1^{5}\right) \quad\left(1^{7}\right)(6,1)$ \\
\hline$(7)(5,2) \quad\left(1^{7}\right)\left(2^{2}, 1^{3}\right)$ & & & 1 & & & & & & & & & & & & $(7)\left(2_{3}^{2} 1^{3}\right) \quad\left(1^{7}\right)(5,2)$ \\
\hline$\overline{(7) \&\left(5,1^{2}\right)} \quad\left(1^{7}\right)\left(3,1^{4}\right)$ & & & & 1 & & & & & & & & & & & $(7)\left(3,2^{4}\right) \quad\left(1^{7}\right)\left(5,1^{2}\right)$ \\
\hline$\left(1^{7} \otimes\left(2^{3}, 1\right)\right.$ & & & & & 1 & & & & & & & & & & $\left(1^{7}\right)(4,3)$ \\
\hline$(7)(4,2,1) \quad\left(1^{7}\right)\left(3,2,1^{2}\right)$ & & & & & & 1 & & & & & & & & & $(7)\left(3,2,1^{2}\right)\left(1^{7}\right)(4,2,1)$ \\
\hline$(7)\left(3^{2} 1\right) \quad\left(2^{7}\right)\left(3,2^{2}\right)$ & & & & & & & 1 & & & & & & & & $\left.(7)\left(3,2^{2}\right) \quad\left(1^{7}\right) \times 3^{2}, 1\right)$ \\
\hline$(7)\left(4,1^{3}\right) \quad\left(1^{7}\right)\left(4,1^{3}\right)$ & & & & & & & & 1 & & & & & & & . \\
\hline$(6,1)(6,1) \quad\left(2,1^{5}\right)\left(2,1^{5}\right)$ & 1 & 1 & 1 & 1 & & & & & & & & & & & $(6,1)\left(2,1^{5}\right)$ \\
\hline$(6,1)(5,2) \quad\left(2,1^{5}\right)\left(2^{2}, 1^{3}\right)$ & & 1 & 1 & 1 & 1 & 1 & & & & & & & & & $(6,1)\left(2^{2} 1^{3}\right)\left(2,1^{5}\right)(5,2)$ \\
\hline$(6,1)\left(5,1^{2}\right) \quad\left(2,1^{5}\right)\left(3,1^{4}\right)$ & & 1 & 1 & 1 & & 1 & & 1 & & & & & & & $(6,1)\left(3,1^{4}\right)\left(2,1^{5}\right)\left(5,1^{2}\right)$ \\
\hline$(6,1)(4,3) \quad\left(2,1^{5}\right)\left(2^{3}, 1\right)$ & & & 1 & & 1 & 1. & 1 & & & & & & & & $(6,1)\left(2^{3}, 1\right)\left(2,1^{5}\right)(4,3)$ \\
\hline$(6,1)(4,2,1)\left(2,1^{5}\right)\left(3,2,1^{2}\right)$ & & & 1 & 1 & 1 & 2 & $1:$ & 1 & 1 & 1 & & & & & $(6,1)\left(3,2,1^{2}\right)\left(2,1^{5}\right)(4,2,1)$ \\
\hline$(6,1)\left(3^{2}, 1\right) \quad\left(2,1^{5}\right)\left(3,2^{2}\right)$ & & & & & 1 & 1 & \pm & & 1 & $I$ & & & & & $(6,1)\left(3,2^{2}\right)\left(2,1^{2}\right)\left(3^{2}, 1\right)$ \\
\hline$\left(6,1 \otimes\left(4,1^{3}\right) \quad\left(2,1^{5}\right)\left(4,1^{3}\right)\right.$ & & & & 1 & & 1 & & 1 & & 1 & & 1 & & & 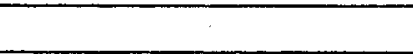 \\
\hline$(5,2)(5,2) \quad\left(2^{2}, 1^{3}\right)\left(2^{2}, 1^{3}\right)$ & 1 & 1 & 2 & 1 & 2 & 2 & 1 & 2 & 1 & & & & & & $(5,2)\left(2^{2}, 1^{3}\right)$ \\
\hline 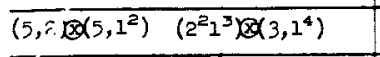 & & 1 & 1 & 2 & 1 & 2 & 2 & 1 & & 1 & & & & & $(5,2)\left(3,1^{4}\right)\left(2^{2}, 1^{3}\right)\left(5,1^{2}\right)$ \\
\hline$\overline{(5}, 2)(4,3) \quad\left(2^{2} 3^{3}\right)\left(2^{3}, 1\right)$ & & 1 & 1 & 1 & 1 & 2 & 1 & & 1 & 1 & & & & & $(5,2)\left(2^{3}, 1\right) \quad\left(2^{2}, 1^{3}\right)(4,3)$ \\
\hline$\left(5,2 \otimes(4,2,1)\left(2^{2} 1^{3}\right) \otimes\left(3,2,1^{2}\right)\right.$ & & 1 & 2 & 2 & 2 & 4 & 2 & 2 & 2 & 3 & 19 & 1 & & & $(5,2)\left(3,2,1^{2}\right) \quad\left(2^{2}, 1^{3}\right)(4,2,1)$ \\
\hline$(5,2)\left(3^{2}, 1\right) \quad\left(2^{2}, 1^{3}\right)\left(3,2^{2}\right)$ & & & 1. & 1 & 1 & 2 & 2 & 1 & 1 & 2 & 1 & 1 & & & $(5,2)\left(3,2^{2}\right) \quad\left(2^{2}, 1^{3}\right) \times\left(3^{2}, 1\right)$ \\
\hline$(5,2)\left(1,1^{3}\right)\left(2^{2} 1^{2}\right)\left(4,1^{3}\right)$ & & & 1 & 1 & & 2 & 1 & 2 & 1 & 2 & & $x$ & & & \\
\hline$\left(5,1^{2}\right) \times\left(5,1^{2}\right)\left(3,1^{4}\right) \in\left(3,1^{4}\right)$ & 1 & 1 & 2 & 1 & 1 & 2 & & 1 & 1 & 1 & & 1 & & & $\left(5,1^{2}\right)\left(3,1^{4}\right)$ \\
\hline$\left(5,1^{2}\right) \times(4,3) \quad\left(3,1^{4}\right) \times\left(2^{3} 1\right)$ & & & 1 & 1 & 1 & 2 & 1 & 1 & 1 & 1 & & & & & $\left(5,1^{2}\right)\left(2^{3} 1\right) \quad\left(3,1^{4}\right)(4,3)$ \\
\hline$\left(5,1^{2}\right) \times(4,2,1)\left(3^{4}\right) \otimes\left(3,2,1^{2}\right)$ & & 1 & 2 & 2 & 2 & 4 & 3 & 2 & 2 & 3 & 1 & 11 & & & $\left(5,1^{2}\right)\left(3,2,1^{2}\right)\left(3,1^{4}\right) \times(1,2,1)$ \\
\hline$\left(5,1^{2}\right) \times\left(3^{2}, 1\right) \quad\left(3,1^{4}\right) \times\left(3,2^{2}\right)$ & & & 3 & & 1 & 3 & 1 & 1 & 2 & 2 & 1 & 1 & & & $\left(5,1^{2}\right) \times\left(3,2^{2}\right) \quad\left(3,1^{4}\right)\left(3^{2}, 1\right)$ \\
\hline$\left(5,1^{2}\right) \times\left(4,1^{3}\right) \quad\left(3,1^{4}\right) \times\left(4,1^{3}\right)$ & & 1 & 1 & 1 & 1 & 2 & 1 & 1 & 1 & 2 & 1 & 12 & 1 & & \\
\hline$(4,3)(4,3) \quad\left(2^{3} 1\right)\left(2^{3} 1\right)$ & 1 & 1 & 1 & 1 & 1 & 1 & 1 & 1 & 1 & 1 & 2 & & & & $(4,3) \times\left(2^{3} 1\right)$ \\
\hline$(4,3)(4,2,1) \quad\left(2^{3} 1\right) \times\left(3,2,1^{2}\right)$ & & 1 & 2 & 2 & 1 & 4 & 2 & 2 & 2 & 3 & 1 & 11 & & & $(4,3) \cup\left(3,2,1^{2}\right) \quad\left(2^{3} 1\right)(4,2,1)$ \\
\hline$(4,3)\left(3^{2}, 1\right) \quad\left(2^{3} 1\right) \times\left(3,2^{2}\right)$ & & 1 & 1 & -1 & 1 & 2 & 1 & 1 & 1 & 2 & 2 & 121 & 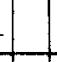 & & $(4,3)\left(3,2^{2}\right) \quad\left(2^{3} 1\right)\left(3^{2}, 1\right)$ \\
\hline$(4,3) \times\left(4,1^{3}\right) \quad\left(2^{3} 1\right)\left(4,1^{3}\right)$ & & & & 1 & 1 & 2 & 1 & 2 & 2 & 2 & 1 & 3 & & & \\
\hline$(4,2,1)(4,2,1)\left(3,2,1^{2}\left(3,2,2^{3}\right)\right.$ & 1 & 2 & 4 & 4 & 4 & 9 & 5 & 5 & 5 & 8 & 3 & 3 & 1 & & $(4,2,1)\left(3,2,2^{2}\right)$ \\
\hline$(4,2,1) \times\left(3^{2}, 1\right) \quad\left(3,2,1^{2}\right)\left(3,2^{2}\right)$ & & 1 & 2 & 3 & 2 & 5 & 3 & 3 & 3 & 5 & 2 & 2 & 1 & & $(4,2,1)\left(3,2^{2}\right)\left(3,2,1^{2}\right)\left(3^{2}, 1\right)$ \\
\hline$(4,2,1)\left(4,1^{3}\right) \quad\left(3,2,1^{2}\right)\left(4,1^{3}\right)$ & & 1 & 2 & 2 & 2. & 5 & 3 & 2 & 3 & 5 & 2 & $2 \longdiv { 2 }$ & 1 & & \\
\hline$\left(3^{2}, 1\right)\left(3^{2}, 1\right) \quad\left(3,2^{2}\right)\left(3,2^{2}\right)$ & 2 & 1 & 2 & 1 & 1 & 3 & 1 & 2 & 2 & 3 & 2 & 21 & 1 & & $\left(3^{2}, 1\right)\left(3,2^{2}\right)$ \\
\hline$\left.\left(3^{2}, 1\right)\left(4,1^{3}\right) \quad\left(3,2^{2}\right) \otimes 4,1^{3}\right)$ & & & 1 & 2 & 1 & 3 & 2 & 2 & 2 & 3 & 1 & 11 & 1 & & \\
\hline$\left(4,1^{3}\right)\left(4,1^{3}\right)$ & 1 & 1 & 2 & 1 & 2 & 2 & $a$ & 1 & 2 & 2 & 2 & 12 & 1 & 1 & \\
\hline & & & & $\left|\begin{array}{l}-5 \\
-5\end{array}\right|$ & 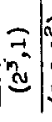 & $\left|\begin{array}{c}0 \\
0 \\
0 \\
\vdots \\
0 \\
0\end{array}\right|$ & त్ & $\begin{array}{l}5 \\
-5 \\
=\end{array}$ & है & $\left|\begin{array}{l}\vec{z} \\
\hat{v} \\
\vec{E}\end{array}\right|$ & $\mid$ & & $\mid \begin{array}{l}3 \\
0 \\
0\end{array}$ & $E$ & \\
\hline
\end{tabular}


TABSE C (CODEt10ued)

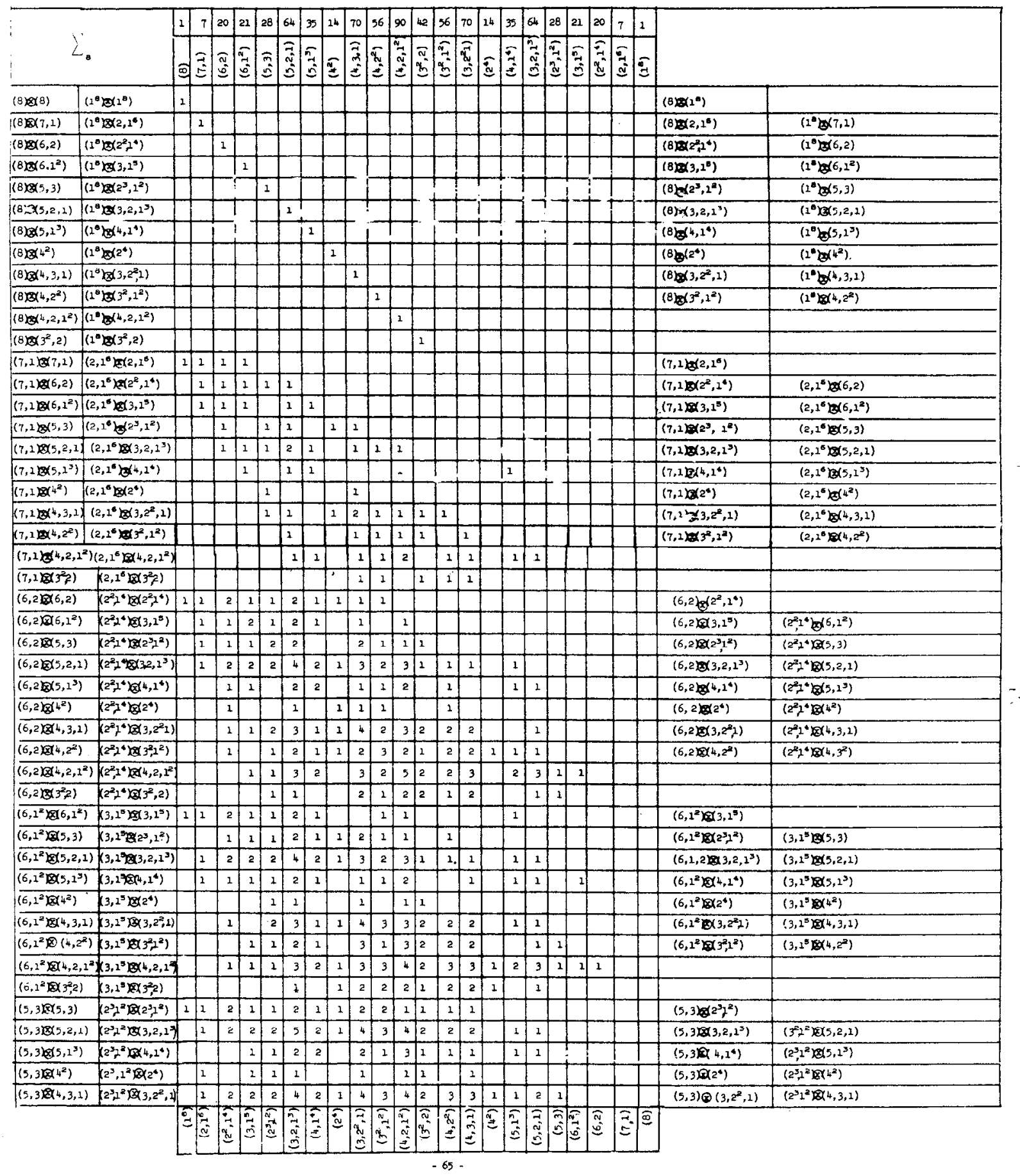




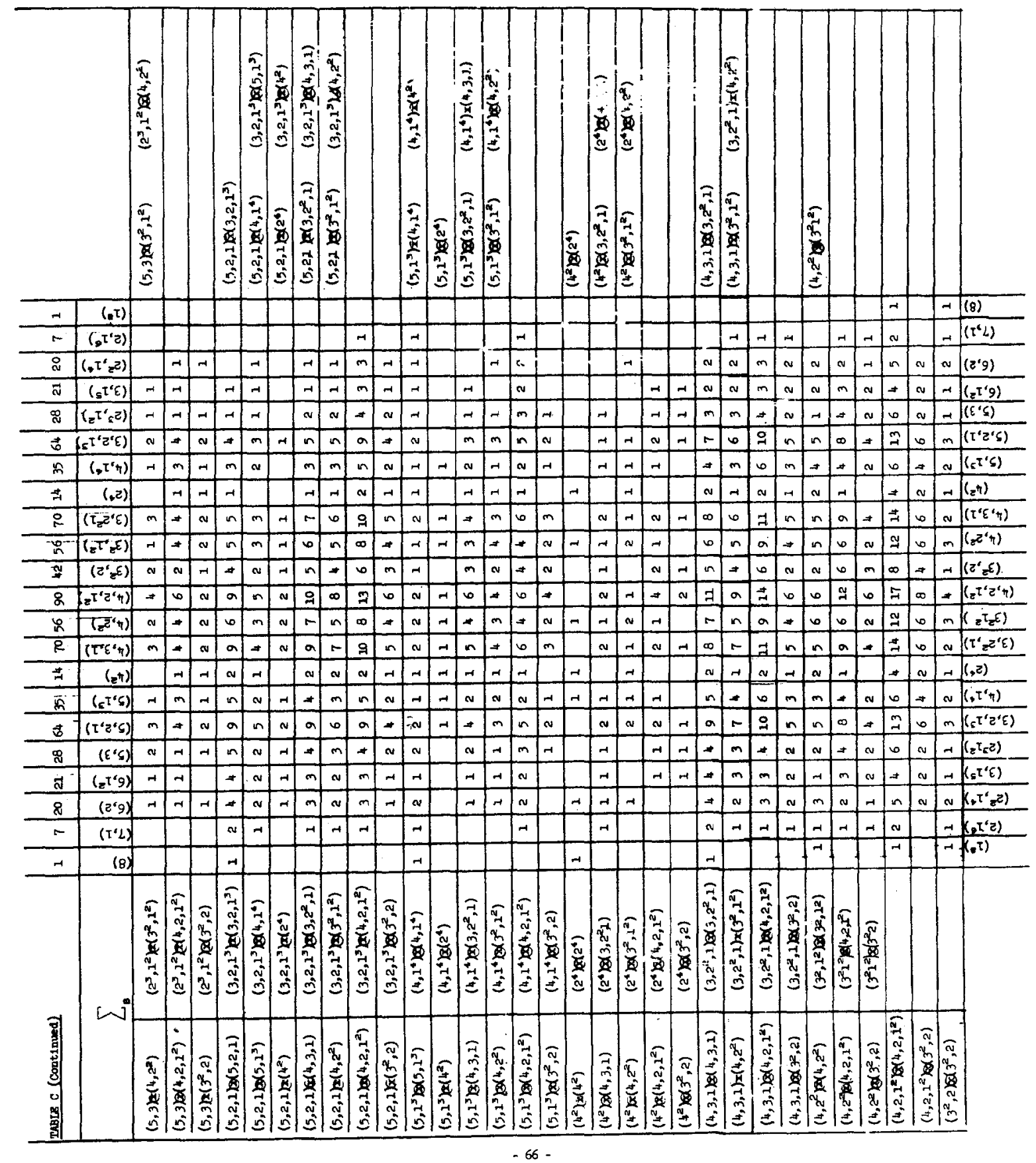


APPENDIX

THE SYMMETRIC GROUP AND PROPERTIES OF THE YOUNG SYMMETRY OPERATORS

The theory of linear groups is intimately linked with the study of the symmetric group $\sum f$, the permutation group of $f$ objects. We have given ir Section III rules for obtaining the ir redraible representations of $S_{n}$ ky imposing certain maximal symetry conditions on the indices of tensors. These symmetry conditions are completely described by the Young tableau. We want to discuss now the fundamental properties of the corresponding Young symmetry operators of the symetric group $\sum f^{*}$

A useful technique for obtailing the irreducible representations of discrete groups is based on the construction of a finite vector space in which the group elements can be chosen as a basis. Such a vector space, in which there exists a natural law of vector multiplication, has the properties of a ring; it is called the group ring. The subspaces of the ring which are left: invariant under this multiplication are called left ideals, and provide representations of the group.

Let $p_{1} p_{2} \cdots p_{g}$ be the elements of a discrete group of $g$ elements. The group ring $R$ is defined by the set of vectors

$$
x=x_{1} p_{1}+x_{2} p_{2}+\cdots+x_{g} p_{g}
$$

where $\left(x_{1} x_{2} \cdots x_{g}\right)$ is a g-uple of complex number, which satisfies the following law of multiplication based on the group multiplication law:

$$
x y \equiv \sum_{i, j} x_{i} y_{j}\left(p_{i} p_{j}\right)=\sum_{h}(x y)_{k} p_{k}
$$

\footnotetext{
* See References 1 to 4.
} 
where

$$
(x y)_{k}=\sum x_{i} y_{j}
$$

and the sum is carried over all $i, j$ for which $p_{i} p_{j}=p_{k}$. A left (right) ideal I is then defined by the condition that if $x \Leftarrow I$, then $y x \Leftarrow I \quad(x y \Leftarrow I)$, for every $y \leqq K$. Two triviel examples of an ideal are the ring $R$, and multiples of the identity.

Due to the associativity of the group multiplication law, it is clear that a left ideal gives rise to a representation of the group. To obtain the irreducible representations we require the minimal ideals, which are those ideals which contain no proper invariunt subspacss. A very important element of an ideal $I$ is its idempotent element $e$, which has the property that $e^{2}=e$, i.e., it is a projection of the ring on the ideal. Suppose that $\mathrm{x} \in \mathrm{R}$; then $\mathrm{xe} \Subset \mathrm{I}$, and if $\mathrm{x} \Leftarrow \mathrm{I}$, then $\mathrm{xe}=\mathrm{x}$. For the permutation group, we want to show that the idempotents of its minimal ideals can be chosen to be precisely the Young symmetry operators $Y$ described earlier in Section III (apart from normalization). The two crucial properties which we have to demonstrate are:

(1) $Y^{2}=\mu Y$ where $\mu$ is a constant;

(2) If $Y=\mu\left(e_{1}+e_{2}\right)$, where $e_{1}^{2}=e_{1}, e_{2}^{2}=e_{2}$ and $e_{1} e_{2}=0$, then either $e_{1}$ or $e_{2}=0$; in other words, the corresponding ideal is minimal.

First we show that if an element $x$ of the permutation ring has the property

$$
x p=x \quad \text { and } \quad q x=\delta_{q} x
$$

where $p$ and $q$ are elements of the Young symmetry operator $Y$ (see Section III, formula (5)), then $\mathrm{x}=\mathrm{cY}$, where $\mathrm{c}$ is a constant. Any element $\mathrm{YZY}$ where 
$z$ belongs to the ring $R$, naturally has this property. Hence $Y^{2}$ must be a multiple of $Y$. To prove property (I) we also have to show that the proportionality constant does not vanish. Finally, the minimal property (2) follows immediately since it also implies that

$$
Y e_{i} Y=e_{i} \quad L-1,2
$$

Hence, by ( 1 ), either $\mu e_{1}=Y, e_{2}=0$ or $e_{1}=0$ and $\mu_{2}=Y$. Expanding $x=\Sigma x(t) t$, we find that the conditions $x p^{-1}=x$ and $q^{-1} x=\delta_{q} x$ imply that $x(t p)=x(t)$ and $x(q t)=\delta_{q} x(t)$, respectively. In particular, substituting for $t$ the ideitity $t=1$, we obtain $x(p)=x(1)$ and $x(q p)=\delta_{q} x(I)$. These are precisely the expansion coefficients of $Y$ (apart from the constant $x(I)$ ). It remains to demonstrate that $x(t)=0$ when $t$ is not a permutation element contained in $Y$. A bit of reflection will show that all permutations which do not belong to $Y$ are characterized by the property that, if they are applied to the integers $I$ to $f$ occupyine the boxes of a Young tableau at least two integers in the same row, end up in the same column. It follows that if $u$ is the transposition of these integers in the initial row and $\mathrm{v}$ is the corresponding transposition in the final column,

$$
v t=t u
$$

But we have the property that

$$
x(t u)=x(t) \quad \text { and } \quad x(v t)=-x(t)
$$

which implies

$$
x(t)=0
$$


Finally, we evaluate the coefficient $\mu$, Eq. (1). For this purpose we introduce a reducible representation for the group generated by the linear transformations induced by the group elements when they act on $R$, the so-called regular representation. The only property of the regular representation which

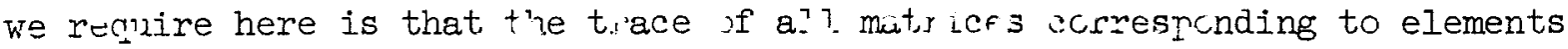
other than the identity vanish, hence trace $Y=f$ (recall that $f$ ! is the dimension of the regular representation, i.e., the order of the symmetric group $\left.\sum_{f}\right)$. On the other hand if we introduce as basis a set of vectors belonging to the ideal generated by $Y$ of dimersion $l, Y$ must be a multiple of the $l \times l$ unit matrix in the corresponding representation. Hence, trace $Y=\mu l$, and $\mu=f ! / \ell$.

We have shown that the Young symmetry operator $Y$ is an idempotent or projection in lhe ring of the symmetric group $\sum_{f}$. It generates a minimal ideal, that is, an invariant subspace under group multiplication which does not contain any smaller invariant subspaces. Hence, it gives an irreducible representation of $\sum f$. In fact, all the irreducible representations of $\sum f$ are given by the possiblc Young tableaux of $f$ boxes. The proof is quite simple and will not be given here. In conclusion, we note that the ideals corresponding to different Young tableaux are carrier spaces for unequivalent representations.

\section{ACKNOWLEDGMENT}

We would like to thank Professors L. Schiff and W.K.H. Panofsky for their hospitality at Stanford University. 
1. H. Weyl, Classical Groups (Princeton University Press, 1946).

2. F. D. Murnaghan, The Theory of Group Representations (Dover 1963).

3. D. Littlewood, Theory of Group Characters and Matrix Representations of Groups (Oxford University Press, 1950).

4. M. Hamermesh: Grcip Throry ara It.3 fopisiatior to Physical Processes (Addison Wesley, 1962).

5. G. Racah, Group Theory and Spectroscopy (Princeton University Press, 1951).

6. M. L. Whippman, "Branching rules for simple Lie groups," University of Pennsylvania preprint (1964).

7. C. R. Hagen and A. J. MacFar Iane, "Reluction of representations of $\mathrm{SU}_{\mathrm{mn}}$ and $\mathrm{SU}_{\mathrm{m}+\mathrm{n}}$ with respect to the subgroup $\mathrm{SU}_{\mathrm{m}}, \mathrm{SU}_{\mathrm{n}}$," Syracuse University preprint (1965). 